\title{
ACTION ON GRASSMANNIANS ASSOCIATED WITH A FIELD EXTENSION
}

\author{
PATRICK RABAU
}

\begin{abstract}
We examine the action of the general linear group $\mathrm{GL}_{L}(V)$ on the set of all $K$-subspaces of $V$, where $L / K$ is a finite field extension and $V$ is a finite-dimensional vector space over $L$. The orbits are completely classified in the case of quadratic and cubic extensions; for infinite fields, the number of orbits is shown to be infinite if the degree of the extension is at least four. As an application we obtain $q$-analogues of tranformation and evaluation formulas for hypergeometric functions due to Gessel and Stanton.
\end{abstract}

\section{INTRODUCTION}

This paper investigates the action on Grassmannians of a general linear group defined over an extension field of the base field in the following context. We have a finite extension of fields $L / K$ and a finite-dimensional vector space $V$ over $L$, and consider the natural action of $\mathrm{GL}_{L}(V)$ on the set $\left[{ }^{V}\right]_{K}$ of all $K$ subspaces of $V$, partially ordered by inclusion. In group theoretic language, this corresponds to looking at double coset spaces of the form $P \backslash \mathrm{GL}_{K}(V) / \mathrm{GL}_{L}(V)$ where $P$ is a maximal parabolic subgroup of $\mathrm{GL}_{K}(V)$. The precise structure of these double coset spaces is of great interest in the theory of automorphic forms, especially for the explicit construction of $L$-functions, as developed in [5]. Understanding the general linear situation of the present paper is also a prerequisite for considering analogous questions for other classical groups (see [8]).

We show that, if the fields $K$ and $L$ are infinite, a necessary and sufficient condition for the quotient poset $\left[{ }^{V}\right]_{K} / \mathrm{GL}_{L}(V)$ to be finite is that $[L: K] \leq 3$. The cases of quadratic and cubic extensions are examined in more detail. To each $K$-subspace of $V$ are attached a certain number ( 2 for quadratic and 4 for cubic extensions) of integer invariants which completely characterize the orbits; the structure of the quotient poset depends only on the dimension of the vector space and not on the fields themselves.

Received by the editors December 12, 1989.

1980 Mathematics Subject Classification (1985 Revision). Primary 15A03, 14L30; Secondary 05A30, 06A99.

This paper constitutes a revised portion of the author's Ph.D. thesis written under Professor Dennis Stanton at the University of Minnesota. 
We dispose of the quadratic case quickly, since we feel many of the results should be known (e.g., Corollary 4.3 appears as an exercise in [4]), even though we have not been able to find an adequate reference for them.

We then concentrate on the cubic case, where the quotient poset $\left[{ }^{V}\right]_{K} / \mathrm{GL}_{L}(V)$ has some very nice combinatorial features. It is shown to be isomorphic to a modification of Young's lattice of partitions inside a $3 \times n$ rectangle. We also spend some time examining the sometimes unexpected algebraic properties of a special type of subspaces which we call the 'triangular' subspaces of $V$. Although we will not elaborate on this here, let us mention that these subspaces occur as 'generic' subspaces in the Grassmannian of $K$ subspaces of $V$ having half the dimension of $V$ (when $\operatorname{dim} V$ is even). As far as we know, these results in the cubic case are completely new.

When the fields are finite, we also compute the size of each orbit for the action of $\mathrm{GL}_{L}(V)$ on $\left[{ }^{V}\right]_{K}$ for quadratic and cubic extensions. In the cubic case this leads to new $q$-analogues of transformation and evaluation formulas for hypergeometric series due to Gessel and Stanton [6].

Finally we should mention the paper [7] that considers the natural generalization of the present situation obtained by replacing the extension field $L$ of $K$ by a finite-dimensional commutative semisimple algebra.

\section{Finiteness RESUlts}

We first introduce some general notation that will be used repeatedly. The multiplicative group of a field $K$ is written $K^{\times}$. If $V$ is a vector space over $K$, $\left[{ }^{V}\right]_{K}$ and $\left[\begin{array}{c}V \\ i\end{array}\right]_{K}$ will denote, respectively, the set of all subspaces and the set of $i$-dimensional subspaces of $V$. The zero subspace is written 0 ; the subspace of $V$ generated by a set $S$ is written $K S$, or $\left\langle v_{1}, \ldots, v_{n}\right\rangle_{K}$ if $S=\left\{v_{1}, \ldots, v_{n}\right\}$. Finally, $\mathbf{N}$ is the set of nonnegative integers, and the set-theoretic difference of two sets is written $S-T$.

Throughout this paper $L / K$ will be a finite extension of fields, and $V$ will be a finite-dimensional vector space over $L$.

Since $V$ is also a vector space over $K$ by restriction of scalars, we have the set $\left[{ }^{V}\right]_{K}$ of $K$-subspaces of $V$, partially ordered by inclusion. In general, given a poset $\mathscr{P}$ of finite height and a group $G$ acting on $\mathscr{P}$ by poset automorphisms, we define the quotient poset $\mathscr{P} / G$ to be the set of orbits for the action of $G$ on $\mathscr{P}$, ordered by $O_{1} \leq O_{2}$ if there exist $x \in O_{1}, y \in O_{2}$ such that $x \leq y$ in $\mathscr{P}$. In our situation, the group $\mathrm{GL}_{L}(V)$ acts by automorphisms on the poset $\left[\begin{array}{l}V \\ *\end{array}\right]_{K}$, and we want to investigate the quotient poset $\left[\begin{array}{l}V \\ *\end{array}\right]_{K} / \mathrm{GL}_{L}(V)$. If $W \in\left[\begin{array}{l}{ }^{V} \\ { }^{*}\end{array}\right]_{K}$, we will use the notation

$$
\operatorname{Stab}_{V}(W)=\left\{T \in \mathrm{GL}_{L}(V) \mid T(W)=W\right\} .
$$

Since the poset $\left[\begin{array}{l}V \\ { }^{V}\end{array}\right]_{K}$ is ranked, so is the quotient poset. The cardinality of the $j$ th level of the quotient poset is the size of the double coset space

$$
P \backslash \mathrm{GL}_{K}(V) / \mathrm{GL}_{L}(V),
$$


where $P$ is some suitable maximal parabolic subgroup of $\mathrm{GL}_{K}(V)$. In terms of matrices, if $\operatorname{dim}_{L} V=n$ and $[L: K]=s$, we have to consider the double coset space

$$
P \backslash \mathrm{GL}(s n, K) / \mathrm{GL}(n, L)
$$

with

$$
P=\left\{\left(\begin{array}{cc}
* & * \\
0_{j \times(s n-j)} & *
\end{array}\right)\right\} \leq \mathrm{GL}(s n, K),
$$

where we have chosen a suitable embedding of $\mathrm{GL}(n, L)$ into $\mathrm{GL}(s n, K)$. We have used right action notation here, but later we mostly use left action. The intended meaning will always be clear.

Our main result is the following:

Theorem 2.1. Let $L / K$ be a finite extension of fields, and let $V$ be a nonzero finite-dimensional vector space over $L$. Suppose the two fields are infinite. Then the quotient poset $\left[\begin{array}{l}V \\ *\end{array}\right]_{K} / \mathrm{GL}_{L}(V)$ is finite if and only if $[L: K] \leq 3$.

Before giving the proof of the theorem we need a lemma whose proof we shall omit.

Lemma 2.2. Let $K$ be an infinite field, and let $F(X, Y)$ be a nonzero polynomial in two variables over $K$. Then there exists an infinite family $\left(a_{i}\right)_{i \in I}$ in $K$ such that for any distinct $i$ and $j, F\left(a_{i}, a_{j}\right) \neq 0$.

Proof of Theorem 2.1. The finiteness of the quotient poset in the case $[L$ : $K] \leq 3$ is shown in $\S \S 4$ and 5. Assume now that $[L: K] \geq 4$. To prove that the quotient poset is infinite, it is enough to show that there are infinitely many orbits for the action of $\mathrm{GL}_{L}(L) \cong L^{\times}$on $\left[\begin{array}{l}L \\ 2\end{array}\right]_{K}$. Pick $\alpha \in L-K$. If $[K(\alpha): K] \leq 3$ and if $\beta$ is an element of $L$ not in $K(\alpha)$, then $1, \alpha, \beta, \alpha \beta$ are independent over $K$. If $[K(\alpha): K] \geq 4$, take $\beta=\alpha^{2}$, so that $1, \alpha, \beta$, $\alpha \beta$ are also independent over $K$.

Consider subspaces of the form $W(a)=\langle 1, a \alpha+\beta\rangle_{K} \in\left[\begin{array}{c}L \\ 2\end{array}\right]_{K}(a \in K)$. Let $a, b \in K$ and suppose $W(a)$ and $W(b)$ are in the same orbit. Then there exists a scalar $\lambda \in L^{\times}$such that $\langle 1, a \alpha+\beta\rangle_{K}=\lambda\langle 1, b \alpha+\beta\rangle_{K}$, which implies

$$
\left\{\begin{array}{l}
\lambda=p+q(a \alpha+\beta) \\
\lambda(b \alpha+\beta)=r+s(a \alpha+\beta)
\end{array}\right.
$$

for some scalars $p, q, r, s$ in $K$, not all zero. Eliminating $\lambda$ between these two equations yields

$$
r+s(a \alpha+\beta)-p(b \alpha+\beta)-q\left(\beta^{2}+\alpha \beta(a+b)+a b \alpha^{2}\right)=0
$$

i.e., the vectors

$$
1, a \alpha+\beta, b \alpha+\beta, \beta^{2}+\alpha \beta(a+b)+a b \alpha^{2}
$$

are linearly dependent over $K$. Complete $\{1, \alpha, \beta, \alpha \beta\}$ to a basis of $L$ over $K$, and take the first four components of each vector in (1) with respect to that basis; the $4 \times 4$ determinant of these components has to be zero. 
Introduce $X$ and $Y$ independent indeterminates and consider the four polynomials

$$
1, \alpha X+\beta, \alpha Y+\beta, \beta^{2}+\alpha \beta(X+Y)+\alpha^{2} X Y
$$

in $L[X, Y]$. Note that the four vectors in (1) are obtained from (2) by substitution of $a$ for $X$ and $b$ for $Y$. Taking the components with respect to $\{1, \alpha, \beta, \alpha \beta\}$, we obtain the matrix

$$
\left(\begin{array}{cccc}
1 & 0 & 0 & * \\
0 & X & Y & * \\
0 & 1 & 1 & * \\
0 & 0 & 0 & (X+Y)+c+d X Y
\end{array}\right)
$$

for some $c, d \in K$. The determinant of this matrix is the nonzero polynomial

$$
F(X, Y)=(X-Y)(X+Y+c+d X Y) \in K[X, Y],
$$

and if $W(a)$ and $W(b)$ are in the same orbit, we necessarily have $F(a, b)=0$.

We may now apply Lemma 2.2 to find an infinite family $\left(a_{i}\right)_{i \in I}$ in $K$ such that, for any distinct $i$ and $j, F\left(a_{i}, a_{j}\right) \neq 0$. Then the subspaces $W\left(a_{i}\right)$ are all in different orbits for the action of $L^{\times}$.

Remark. The remaining sections of the paper actually show a much stronger result. If $[L: K] \leq 3$, the quotient poset $\left[\begin{array}{l}V \\ *\end{array}\right]_{K} / \mathrm{GL}_{L}(V)$ is independent of the fields $K$ and $L$ and only depends on the degree $[L: K]$ and the dimension $\operatorname{dim}_{L} V$.

In the rest of this section, we state a few more general facts about the quotient poset $\left[{ }^{V}\right]_{K} / \mathrm{GL}_{L}(V)$, beginning with a duality result. We do not assume $[L: K] \leq 3$.

Theorem 2.3. The quotient poset $\left[{ }^{V}\right]_{K} / \mathrm{GL}_{L}(V)$ admits an involutory antiautomorphism. In particular, if it is finite, it is rank-symmetric.

Proof. Let $B: V \times V \rightarrow L$ be a nondegenerate symmetric $L$-bilinear form and let $\phi: L \rightarrow K$ be a nonzero $K$-linear map. The map $B^{\prime}=\phi \circ B$ is a nondegenerate symmetric $K$-bilinear form $V \times V \rightarrow K$. For $W \in\left[{ }^{V}{ }^{*}\right]_{K}$, let $W^{\perp}$ denote the orthogonal of $W$ with respect to $B^{\prime}$. The correspondence $W \mapsto W^{\perp}$ is an involutory antiautomorphism of $\left[\begin{array}{l}V \\ *\end{array}\right]_{K}$. Denote the transpose of an endomorphism $T \in \operatorname{End}_{L}(V)$ with respect to $B$ by ${ }^{t} T$, i.e., $B(T u, v)=$ $B\left(u,{ }^{t} T v\right)$ for $u, v \in V$. One checks that $(T W)^{\perp}={ }^{t}\left(T^{-1}\right)\left(W^{\perp}\right)$ for $W \in$ $\left[{ }^{V}{ }^{*}\right]_{K}$ and $T \in \mathrm{GL}_{L}(V)$. Therefore the correspondence $W \mapsto W^{\perp}$ induces an involutory antiautomorphism of the quotient poset $\left[\begin{array}{l}V \\ *\end{array}\right]_{K} / \mathrm{GL}_{L}(V)$.

The fact that the quotient poset is rank-symmetric if it is finite is also contained in the following result. Recall that a finite ranked poset is called Peck if it is rank-symmetric, rank-unimodal and strongly Sperner (see [10] for details).

Theorem 2.4. If the poset $\left[{ }^{V}\right]_{K} / \mathrm{GL}_{L}(V)$ is finite, then it is Peck. 
Proof. If $K$ is a finite field, then $\left[\begin{array}{l}V \\ *\end{array}\right]_{K}$ is unitary Peck [10, Theorem 2]. The poset $\left[\begin{array}{l}V \\ { }^{*}\end{array}\right]_{K} / \mathrm{GL}_{L}(V)$ is then Peck as quotient of a unitary Peck poset [10, Theorem 1]. If $K$ is infinite but $\left[{ }^{V}\right]_{K} / \mathrm{GL}_{L}(V)$ is finite, Theorem 2.1 and the remark following it show that $\left[\begin{array}{l}V \\ *\end{array}\right]_{K} / \mathrm{GL}_{L}(V)$ is isomorphic to a quotient poset of the same form with $K$ finite. So the result is also true in this case.

\section{BASIC RESULTS}

In this section we introduce more terminology and develop some basic results in the context of a field extension $L / K$ of arbitrary degree. Most of these will be useful to us when we examine in detail the cases of quadratic and cubic extensions in subsequent sections.

For a $K$-subspace $W$ of $V$, we write $\left[\begin{array}{l}W \\ *\end{array}\right]_{L}$ for the set of $L$-subspaces of $V$ contained in $W$. For $K$-subspaces $W, W_{1}, \ldots, W_{m}$ of $V$, write

$$
W=W_{1} \oplus_{L} \cdots \oplus_{L} W_{m}
$$

if $W=\bigoplus_{i} W_{i}$ and $L W=\bigoplus_{i} L W_{i}$, in which case we say that the sum $\bigoplus_{i} W_{i}$ is a direct sum over $L$. For two summands we have the equivalent formulation: $W=W_{1} \oplus_{L} W_{2}$ if and only if $W=W_{1} \oplus W_{2}$ and $L W_{1} \cap L W_{2}=0$.

3.1. $L$-component of a subspace. For $W \in\left[{ }^{V}\right]_{K}$, the largest $L$-subspace of $V$ contained in $W$ will be called the $L$-component of $W$ and written $\operatorname{comp}_{L} W$. So $\operatorname{comp}_{L} W=\bigcap_{\lambda \in L^{\times}} \lambda W$ and for a vector $v \in V$, we have

$$
v \in \operatorname{comp}_{L} W \Leftrightarrow L v \subseteq W .
$$

Remark. Let $W \in\left[\begin{array}{c}V \\ *\end{array}\right]_{K}$ and $U \in\left[\begin{array}{l}W \\ { }^{*}\end{array}\right]_{L}$. Viewing the quotient space $W / U$ as a $K$-subspace of the $L$-space $V / U$, one easily checks that $\operatorname{comp}_{L}(W / U)=$ $\left(\operatorname{comp}_{L} W\right) / U$. In particular, $\operatorname{comp}_{L}\left(W / \operatorname{comp}_{L} W\right)=0$.

The following elementary leinma compares the behavior of taking the $L$-span and the $L$-component with respect to sum and intersection of subspaces.

Lemma 3.1. Let $W_{1}, W_{2} \in\left[\begin{array}{l}V \\ *\end{array}\right]_{K}$. Then

(i) $L\left(W_{1}+W_{2}\right)=L W_{1}+L W_{2}$;

(ii) $\operatorname{comp}_{L}\left(W_{1} \cap W_{2}\right)=\operatorname{comp}_{L} W_{1} \cap \operatorname{comp}_{L} W_{2}$;

(iii) $L\left(W_{1} \cap W_{2}\right) \leq L W_{1} \cap L W_{2}$;

(iv) $\operatorname{comp}_{L}\left(W_{1}+W_{2}\right) \geq \operatorname{comp}_{L} W_{1}+\operatorname{comp}_{L} W_{2}$, with equality if the sum $W_{1}+W_{2}$ is direct over $L$.

Proposition 3.2. Let $W \in\left[\begin{array}{l}V \\ *\end{array}\right]_{K}$. If $\left\{\eta_{1}, \ldots, \eta_{s}\right\}$ is a basis of $L$ over $K$, then

$$
L W=\eta_{1} W+\cdots+\eta_{s} W
$$

and

$$
\operatorname{comp}_{L} W=\eta_{1}^{-1} W \cap \cdots \cap \eta_{s}^{-1} W .
$$

In particular, if $L=K(\eta)$ for some $\eta \in L-K$ and $s=[L: K]$, then

$$
\operatorname{comp}_{L} W=W \cap \eta W \cap \cdots \cap \eta^{s-1} W .
$$


Proposition 3.3. Let $W \in\left[\begin{array}{l}V \\ *\end{array}\right]_{K}$ and $U \in\left[\begin{array}{l}W \\ { }_{*}\end{array}\right]_{L}$. Then there exists a subspace $Z \in\left[\begin{array}{c}W \\ { }^{*}\end{array}\right]_{K}$ such that $W=U \oplus_{L} Z$. For any other subspace $Z^{\prime}$ such that $W=$ $U \oplus_{L} Z^{\prime}$, there exists $T \in \mathrm{GL}_{L}(V)$ fixing $U$ pointwise such that $T(Z)=Z^{\prime}$. In particular, $Z$ and $Z^{\prime}$ are in the same orbit for $\mathrm{GL}_{L}(V)$.

Proof. Choose $V_{1} \in\left[\begin{array}{l}V \\ { }^{*}\end{array}\right]_{L}$ such that $L W=U \oplus V_{1}$ and put $Z=V_{1} \cap W$. From the fact that $U \leq W$, one obtains $W=U \oplus Z, L Z=V_{1}$ and $U \cap L Z=$ $U \cap V_{1}=0$, i.e., $W=U \oplus_{L} Z$. We leave the rest of the proof to the reader.

Remark. In general, an arbitrary complement of $U$ in $W$ over $K$ will not form a direct sum over $L$ with $U$. Examples are easy to find, already in the quadratic extension case. However, Theorem 4.1(c) will show that, in the case of quadratic extensions, if $U$ is a $K$-subspace of $W$ containing $\operatorname{comp}_{L} W$, then any complement of $U$ in $W$ over $K$ is a complement over $L$. This last result is not true for cubic or higher extensions, even if $U=\operatorname{comp}_{L} W$.

3.2. Type of a vector in a subspace. The following terminology will be of convenient use. For a subspace $W \in\left[\begin{array}{l}V \\ { }^{*}\end{array}\right]_{K}$ and a vector $v \in W$, we define the type of $v$ in $W$ by

$$
\operatorname{type}_{W}(v)=\operatorname{dim}_{K}(W \cap L v) .
$$

So type ${ }_{W}(0)=0$ and if $v \neq 0$, type ${ }_{W}(v)=\operatorname{dim}_{K}\{\lambda \in L \mid \lambda v \in W\}$. Often we will just talk about the 'type of $v$ ' without specifying the subspace $W$ if it is clear from the context. Note that we define the type of $v$ in $W$ only for vectors of $W$. The vectors $v$ for which $\operatorname{type}_{W}(v)=[L: K]$ are the nonzero vectors in $\operatorname{comp}_{L} W$.

The next lemma shows how the type of vectors behaves with respect to a direct sum over $L$.

Lemma 3.4. Let $W, W_{1}, W_{2} \in\left[\begin{array}{l}V \\ *\end{array}\right]_{K}$ with $W=W_{1} \oplus_{L} W_{2}$. Suppose $v=$ $v_{1}+v_{2} \in W$ with $0 \neq v_{i} \in W_{i}(i=1,2)$. Then

(i) $\operatorname{type}_{W}(v) \leq \min \left(\operatorname{type}_{W_{1}}\left(v_{1}\right), \operatorname{type}_{W_{2}}\left(v_{2}\right)\right)$.

(ii) If $\operatorname{type}_{W_{1}}\left(v_{1}\right)=[L: K]$, then $\operatorname{type}_{W}(v)=\operatorname{type}_{W_{2}}\left(v_{2}\right)$.

Proof. For (i), use the fact that for $\lambda \in L, \lambda v \in W$ if and only if $\lambda v_{i} \in$ $W_{i} \quad(i=1,2)$. For (ii), if type $W_{1}\left(v_{1}\right)=[L: K]$, then $\lambda v_{1} \in W_{1}$ is always satisfied, so that $\lambda v \in W$ if and only if $\lambda v_{2} \in W_{2}$.

Lemma 3.5. Put $s=[L: K]$. The group $L^{\times}$acts (by multiplication) transitively on $\left[\begin{array}{c}L \\ s-1\end{array}\right]_{K}$.

Proof. Take two hyperplanes $H_{1}, H_{2} \in\left[\begin{array}{c}L \\ s-1\end{array}\right]_{K}$ and let $\alpha_{1}, \ldots, \alpha_{s-1}$ be a basis of $H_{1}$. Since $H_{2}$ is a hyperplane of $L, W=\bigcap_{i=1}^{s-1} \alpha_{i}^{-1} H_{2} \neq 0$. Any nonzero scalar $\lambda \in W$ satisfies $\lambda \alpha_{i} \in H_{2}$ for all $\vdots$, i.e., $\lambda H_{1} \leq H_{2}$, which is equivalent to $\lambda H_{1}=H_{2}$.

Corollary 3.6. Fix a basis $\left\{\alpha_{1}, \ldots, \alpha_{s}\right\}$ of $L$ over $K$. Let $v \in W \in\left[\begin{array}{l}V \\ *\end{array}\right]_{K}$. If $\operatorname{type}_{W}(v)=s-1$, then $L v \cap W=\left\langle\alpha_{1} v^{\prime}, \ldots, \alpha_{s-1} v^{\prime}\right\rangle_{K}$ for some vector $v^{\prime}$. 
3.3. $K$-substructures. If $W \in\left[\begin{array}{l}V \\ *\end{array}\right]_{K}$, it is easy to see that

$$
\operatorname{dim}_{L} L W \leq \operatorname{dim}_{K} W \leq s \operatorname{dim}_{L} L W,
$$

where $s=[L: K]$. Equality occurs in the second inequality exactly when $W$ is an $L$-subspace of $V$. We want to single out the subspaces for which equality occurs in the first inequality; these subspaces will play a useful role for us in the sequel. They are characterized by the following lemma, whose proof will be left to the reader (or see [4, II $\S 8]$ ).

Lemma 3.7. Let $\left.W \in\left[{ }^{V}\right]_{K}\right]_{K}$. The following conditions are equivalent:

(i) $\operatorname{dim}_{L} L W=\operatorname{dim}_{K} W$.

(ii) Any $K$-independent subset of $W$ is L-independent.

(iii) Some basis of $W$ over $K$ is L-independent.

(iv) The L-linear map $W \otimes_{K} L \rightarrow V$ induced by the inclusion $W \subseteq V$ is an injection.

Subspaces $W$ satisfying the conditions of the lemma will be called $K$ substructures of $V$. Following Borel [3], $K$-substructures of maximum dimension, i.e., for which $V=L W$, will be called $K$-structures. We refer to [9] for more details about the combinatorics of $K$-substructures.

Remarks. (a) Let $\left\{\eta_{1}, \ldots, \eta_{s}\right\}$ be a basis of $L$ over $K$. A subspace $W \in\left[\begin{array}{l}V \\ *\end{array}\right]_{K}$ is a $K$-substructure if and only if $L W=\eta_{1} W \oplus \cdots \oplus \eta_{s} W$.

(b) If $W$ is a $K$-substructure of $V$, then every nonzero vector of $W$ has type 1 in $W$. We will see later that the converse is true in the case of quadratic extensions, but not for cubic or higher extensions.

The following result is standard and will be useful to us when we consider the case of cubic extensions.

Proposition 3.8. Let $Z$ be a $K$-structure of $V$. Let $V^{\prime}$ be another vector space over $L$ and let $W^{\prime} \in\left[V^{\prime}{ }^{*}\right]_{K}$.

(a) Any map $T \in \operatorname{Hom}_{K}\left(Z, V^{\prime}\right)$ has a unique extension to a map $\widetilde{T} \in$ $\operatorname{Hom}_{L}\left(V, V^{\prime}\right)$. The correspondence $T \mapsto \widetilde{T}$ furnishes a bijection between $\operatorname{Hom}_{K}\left(Z, W^{\prime}\right)$ and $\left\{S \in \operatorname{Hom}_{L}\left(V, V^{\prime}\right) \mid S(Z) \leq W^{\prime}\right\}$, whose inverse is given by the restriction map $\left.S \mapsto S\right|_{Z}$.

(b) In the case where $V=V^{\prime}$, the correspondence $T \mapsto \widetilde{T}$ is an isomorphism between the rings $\operatorname{End}_{K}(Z)$ and $\left\{S \in \operatorname{End}_{L}(V) \mid S(Z) \leq Z\right\}$, which restricts to an isomorphism between the groups $\mathrm{GL}_{K}(Z)$ and $\operatorname{Stab}_{V}(Z)$.

Proposition 3.9. Let $Z$ be a $K$-structure of $V$. Let $V^{\prime}$ be another vector space over L. Let $T \in \operatorname{Hom}_{K}\left(Z, V^{\prime}\right)$ and let $\tilde{T} \in \operatorname{Hom}_{L}\left(V, V^{\prime}\right)$ be its unique $L$-linear extension to $V$.

(a) $\operatorname{dim}_{L} \operatorname{ker} \widetilde{T}=\operatorname{dim}_{K} \operatorname{ker} T+\left(\operatorname{dim}_{K} T(Z)-\operatorname{dim}_{L} L T(Z)\right)$.

(b) The following conditions are equivalent:

(i) $\widetilde{T}$ is injective.

(ii) $\operatorname{dim}_{L} L T(Z)=\operatorname{dim}_{K} Z$.

(iii) $T$ is injective and $T(Z)$ is a $K$-substructure. 
Proof. Let $T$ and $\widetilde{T}$ be as above. The domain of $\widetilde{T}$ has dimension $\operatorname{dim}_{L} V=$ $\operatorname{dim}_{K} Z$ and the image of $\widetilde{T}$ equals $L T(Z)$. Therefore

$$
\begin{aligned}
\operatorname{dim}_{L} \operatorname{ker} \widetilde{T} & =\operatorname{dim}_{K} Z-\operatorname{dim}_{L} L T(Z) \\
& =\left(\operatorname{dim}_{K} Z-\operatorname{dim}_{K} T(Z)\right)+\left(\operatorname{dim}_{K} T(Z)-\operatorname{dim}_{L} L T(Z)\right) \\
& =\operatorname{dim}_{K} \operatorname{ker} T+\left(\operatorname{dim}_{K} T(Z)-\operatorname{dim}_{L} L T(Z)\right),
\end{aligned}
$$

which proves (a) as well as the equivalence of the three conditions in (b).

\section{QuADRATIC EXTENSIONS}

Throughout this section, $L$ is a quadratic extension of $K$ and $V$ is a vector space of finite dimension $n$ over $L$. We fix an element $\eta \in L$ such that $L=K(\eta)$.

\subsection{The general case.}

Theorem 4.1. Let $W \in\left[\begin{array}{l}V \\ *\end{array}\right]_{K}$.

(a) $\operatorname{comp}_{L} W=W \cap \eta W$ and

$$
\operatorname{dim}_{L} \operatorname{comp}_{L} W=\operatorname{dim}_{K} W-\operatorname{dim}_{L} L W .
$$

For $x \in W$, we have $x \in \operatorname{comp}_{L} W \Leftrightarrow \eta x \in W$.

(b) $W$ is a $K$-substructure $\Leftrightarrow \operatorname{comp}_{L} W=0 \Leftrightarrow$ every nonzero vector of $W$ has type 1 in $W$.

(c) Suppose $W=U \oplus_{L} Y$ for some $K$-subspaces $U, Y$ with $\operatorname{comp}_{L} W \leq$ $U$. Then $Y$ is a $K$-substructure, $W=U \oplus_{L} Y$ and $\operatorname{dim}_{K} Y=$ $\operatorname{dim}_{L}(L W / L U)$.

(d) Let $U \in\left[{ }^{W}\right]_{K}$ satisfy $U=W \cap L U$ (equivalently, $U=W \cap V_{1}$ for some $\left.V_{1} \in\left[\begin{array}{l}V \\ *\end{array}\right]_{L}\right)$. Then there exists a $K$-subspace $Z$ such that $W=$ $U \oplus_{L} Z$.

Note that (d) extends Proposition 3.3; this extension does not hold when $[L: K] \geq 3$. Also (c) justifies the Remark following Proposition 3.3.

Proof. (a) Proposition 3.2 implies $\operatorname{comp}_{L} W=W \cap \eta W$. Using the fact that $L W=W+\eta W$, one easily checks formula (3).

(b) The first equivalence follows from (3) and the definition of $K$-substructure. For the second equivalence, use the fact that the vectors of type 2 in $W$ are exactly the nonzero vectors in $\operatorname{comp}_{L} W$.

(c) Clearly $\operatorname{comp}_{L} Y=0$; so $Y$ is a $K$-substructure by (b). To prove the sum $U \oplus Y$ is direct over $L$, we have to show $L U \cap L Y=0$. Let $x \in L U \cap L Y$, and say $x=y_{1}+\eta y_{2}=u_{1}+\eta u_{2}$ with $y_{1}, y_{2} \in Y$ and $u_{1}, u_{2} \in U$. Then $\eta\left(y_{2}-u_{2}\right)=u_{1}-y_{1} \in W$ so that, by part (a), $y_{2}-u_{2} \in \operatorname{comp}_{L} W \leq U$. Then $y_{2} \in U \cap Y=0$ and $\eta u_{2} \in \operatorname{comp}_{L} W \leq U$; hence $y_{1}=u_{1}+\eta u_{2} \in U \cap Y=0$ and $x=0$. The last assertion is then clear.

(d) The fact that $U=W \cap L U$ is equivalent to $U=W \cap V_{1}$ for some $V_{1} \in\left[\begin{array}{l}V \\ { }^{*}\end{array}\right]_{L}$ is straightforward to check. Now choose $X \in\left[\begin{array}{l}U \\ *\end{array}\right]_{K}$ such that 
$U=\operatorname{comp}_{L} U \oplus_{L} X$; choose $Y \in\left[\begin{array}{l}V \\ { }_{*}\end{array}\right]_{L}$ such that $\operatorname{comp}_{L} W=\operatorname{comp}_{L} U \oplus Y$. The condition $U=W \cap L U$ implies $\operatorname{comp}_{L} U \leq \operatorname{comp}_{L} W \cap U \leq \operatorname{comp}_{L} W \cap$ $L U=\operatorname{comp}_{L}(W \cap L U)=\operatorname{comp}_{L} U$. Hence $\operatorname{comp}_{L} W \cap U=\operatorname{comp}_{L} U$ and the sum comp $U \oplus X \oplus Y$ is direct. Choose a $K$-subspace $X_{1}$ such that $W=$ $\operatorname{comp}_{L} U \oplus X \oplus Y \oplus X_{1}=\operatorname{comp}_{L} W \oplus X \oplus X_{1}$. Then (c) implies $X \oplus X_{1}$ is a $K$-substructure and $W=\operatorname{comp}_{L} W \oplus_{L} X \oplus_{L} X_{1}=\operatorname{comp}_{L} U \oplus_{L} Y \oplus_{L} X \oplus_{L} X_{1}$. So we may take $Z=X \oplus X_{1}$.

Remark. In the case of a quadratic extension, Proposition 3.9(a) specializes as follows (use equation (3)):

$$
\operatorname{dim}_{L} \operatorname{ker} \widetilde{T}=\operatorname{dim}_{K} \operatorname{ker} T+\operatorname{dim}_{L} \operatorname{comp}_{L} T(Z) .
$$

Define the type of a $K$-subspace $W$ of $V$ to be the ordered pair of nonnegative integers

$$
\operatorname{type}(W)=\left(\operatorname{dim}_{L} \operatorname{comp}_{L} W, \operatorname{dim}_{K}\left(W / \operatorname{comp}_{L} W\right)\right) .
$$

The parameters in type $(W)$ are clearly invariant under the action of $\mathrm{GL}_{L} V$ on $\left[\begin{array}{l}V \\ { }_{*}\end{array}\right]_{K}$. We will see that they form a complete set of invariants for this action.

Theorem 4.2. Let $W \in\left[\begin{array}{l}V \\ { }^{*}\end{array}\right]_{K}$ with type $(W)=(\alpha, t)$. Then there exist $L$ independent vectors $v_{1}, \ldots, v_{\alpha} ; z_{1}, \ldots, z_{t}$ such that

$$
W=\left\langle v_{1}, \ldots, v_{\alpha}\right\rangle_{L} \oplus_{L}\left\langle z_{1}, \ldots, z_{t}\right\rangle_{K} .
$$

Proof. Choose $Y \in\left[\begin{array}{c}W \\ t\end{array}\right]_{K}$ such that $W=\operatorname{comp}_{L} W \oplus_{L} Y$. Pick a basis $v_{1}, \ldots, v_{\alpha}$ of $\operatorname{comp}_{L} W$ over $L$ and a basis $z_{1}, \ldots, z_{t}$ of $Y$ over $K$, and apply Theorem $4.1(\mathrm{c})$.

Corollary 4.3. Two $K$-subspaces of $V$ are in the same orbit for $\mathrm{GL}_{L}(V)$ if and only if they have the same type.

Proof. We have already noticed that the type of a subspace is invariant under the action of $\mathrm{GL}_{L}(V)$. Conversely, if two $K$-subspaces $W$ and $W^{\prime}$ of $V$ have the same type, they can be written in the same standard form given in Theorem 4.2 with respect to suitable bases $\mathscr{B}$ and $\mathscr{B}^{\prime}$. The map in $\mathrm{GL}_{L}(V)$ that sends $\mathscr{B}$ to $\mathscr{B}^{\prime}$ will send $W$ onto $W^{\prime}$.

If type $(W)=(\alpha, t)$, then

$$
\operatorname{dim}_{K} W=2 \alpha+t \text { and } \operatorname{dim}_{L} L W=\alpha+t .
$$

We see that an ordered pair $(\alpha, t) \in \mathrm{N}^{2}$ is the type of a $K$-subspace of $V$ if and only if $\alpha+t \leq n$. Moreover, suppose $\alpha, \alpha^{\prime}, t, t^{\prime} \in \mathbf{N}$ with $\alpha+t \leq n$, 
$\alpha^{\prime}+t^{\prime} \leq n$. Then there exist subspaces $W, W^{\prime} \in\left[\begin{array}{l}V \\ *\end{array}\right]_{K}$ satisfying type $(W)=$ $(\alpha, t), \operatorname{type}\left(W^{\prime}\right)=\left(\alpha^{\prime}, t^{\prime}\right)$ and $W \leq W^{\prime}$ if and only if

$$
\alpha \leq \alpha^{\prime} \text { and } \alpha+t \leq \alpha^{\prime}+t^{\prime} \text {. }
$$

(Use the fact: $W \leq W^{\prime} \Rightarrow \operatorname{comp}_{L} W \leq \operatorname{comp}_{L} W^{\prime}$ and $L W \leq L W^{\prime}$.) The correspondence $(\alpha, t) \mapsto(\alpha+t, t)$ establishes a bijection between $\{(\alpha, t) \in$ $\left.\mathrm{N}^{2} \mid \alpha+t \leq n\right\}$ and the set of partitions inside a $2 \times n$ rectangle. The order relation on ordered pairs defined by (4) corresponds exactly to the usual order relation between partitions (by containment of their Ferrers diagrams). This proves the main result of this section.

Theorem 4.4. Let $L / K$ be a quadratic extension of fields, and let $V$ be a vector space of finite dimension $n$ over $L$. The quotient poset $\left[{ }^{V}\right]_{K} / \mathrm{GL}_{L}(V)$ is finite and independent of the fields $K$ and $L$. It is isomorphic to Young's lattice of partitions inside a $2 \times n$ rectangle.

Remark. We mention the following consequence for applications. The $j$ th level of the quotient poset has size $\lfloor j / 2\rfloor+1$ for $0 \leq j \leq n$ and $\lfloor(2 n-j) / 2\rfloor$ +1 for $n \leq j \leq 2 n$.

Remark. The antiautomorphism of Theorem 2.3 is the involution that sends the type $(\alpha, t)$ to $(n-\alpha-t, t)$, i.e., the involution that sends a partition to its 'complement' in the $2 \times n$ rectangle.

4.2. The finite field case. In the case of finite fields, we can also compute the sizes of the orbits for the action of $\mathrm{GL}_{L}(V)$ on $\left[\begin{array}{l}V \\ { }_{*}\end{array}\right]_{K}$. We shall use the standard abbreviations

$$
\begin{gathered}
(a ; q)_{\infty}=\prod_{i=0}^{\infty}\left(1-a q^{i}\right), \\
(a ; q)_{m}=(a ; q)_{\infty} /\left(a q^{m} ; q\right)_{\infty}, \\
{\left[\begin{array}{c}
m \\
p
\end{array}\right]_{q}=\frac{(q ; q)_{m}}{(q ; q)_{p}(q ; q)_{m-p}}}
\end{gathered}
$$

and

$$
\left[\begin{array}{c}
m \\
p_{1}, \ldots, p_{s}
\end{array}\right]_{q}=\frac{(q ; q)_{m}}{(q ; q)_{p_{1}} \cdots(q ; q)_{p_{s}}} \quad \text { with } m=p_{1}+\cdots+p_{s} \text {. }
$$

Theorem 4.5. Let $K=\mathbf{F}_{q} \leq L=\mathbf{F}_{q^{2}}$, where $q$ is a power of a prime. Let $V$ be a vector space of finite dimension $n$ over $L$. Let $\alpha, t \in \mathbf{N}$ with $\alpha+t \leq n$. The number of $K$-subspaces of $V$ of type $(\alpha, t)$ equals

$$
\begin{aligned}
a(n, \alpha, t) & =\left[\begin{array}{c}
n \\
\alpha, t, n-\alpha-t
\end{array}\right]_{q^{2}}(-q ; q)_{t} q^{\left(\begin{array}{l}
t \\
2
\end{array}\right)} \\
& =\frac{\left(q^{2} ; q^{2}\right)_{n} q^{\left(\begin{array}{c}
t \\
2
\end{array}\right)}}{\left(q^{2} ; q^{2}\right)_{\alpha}\left(q^{2} ; q^{2}\right)_{n-\alpha-t}(q ; q)_{t}},
\end{aligned}
$$


which is a polynomial in $q$ of degree $2 n(\alpha+t)-t^{2}-2 \alpha t-2 \alpha^{2}$ with nonnegative integral coefficients.

Proof. To determine the number $a(n, 0, n)$ of $K$-structures of $V$, count the number of ways to choose an $L$-basis $v_{1}, \ldots, v_{n}$ of $V$ and divide by the number of choices giving the same subspace $\left\langle v_{1}, \ldots, v_{n}\right\rangle_{K}$ to get

$$
\frac{\left(q^{2 n}-1\right)\left(q^{2 n}-q^{2}\right) \cdots\left(q^{2 n}-q^{2 n-2}\right)}{\left(q^{n}-1\right)\left(q^{n}-q\right) \cdots\left(q^{n}-q^{n-1}\right)}=(-q ; q)_{n} q^{\left(\begin{array}{c}
n \\
2
\end{array}\right)} \text {. }
$$

Now count the number of subspaces $W \in\left[\begin{array}{l}V \\ { }^{V}\end{array}\right]_{K}$ of type $(\alpha, t)$ in three steps. Choose $V_{1}=L W$ in $\left[\begin{array}{c}n \\ \alpha+t\end{array}\right]_{q^{2}}$ ways. Inside $V_{1}$ choose $U=\operatorname{comp}_{L} W$ in $\left[\begin{array}{c}\alpha+t \\ \alpha\end{array}\right]_{q^{2}}$ ways. Then, with $U$ and $V_{1}$ fixed, we have to determine the cardinality of the set $\mathscr{A}=\left\{W \in\left[\begin{array}{l}V_{1} \\ *\end{array}\right]_{K} \mid \operatorname{type}(W)=(\alpha, t), \operatorname{comp}_{L} W=U\right\}$. Because $\operatorname{comp}_{L}\left(W / \operatorname{comp}_{L} W\right)=0$, the canonical $L$-linear projection of $V$ onto $V / U$ induces a bijection between $\mathscr{A}$ and $\mathscr{A}^{\prime}=\left\{\bar{W} \in\left[{ }^{V_{1} / U}\right]_{K} \mid\right.$ type $\left.(\bar{W})^{-}=(0, t)\right\}$. Since $\operatorname{dim}_{L}\left(V_{1} / U\right)=t,\left|\mathscr{A}^{\prime}\right|=a(t, 0, t)=(-q ; q)_{t} q^{\left(\frac{1}{2}\right)}$. So

$$
a(n, \alpha, t)=\left[\begin{array}{c}
n \\
\alpha+t
\end{array}\right]_{q^{2}}\left[\begin{array}{c}
\alpha+t \\
\alpha
\end{array}\right]_{q^{2}}(-q ; q)_{t} q^{\left(\frac{t}{2}\right)} .
$$

An elementary computation gives the remaining assertions.

Remark. The generating function for the numbers $a(n, \alpha, t)$ is given by

$$
\sum_{n, \alpha, t \geq 0} \frac{a(n, \alpha, t) x^{n} y^{\alpha} z^{t}}{\left(q^{2} ; q^{2}\right)_{n}}=\frac{(-x z ; q)_{\infty}}{\left(x ; q^{2}\right)_{\infty}\left(x y ; q^{2}\right)_{\infty}} .
$$

\section{Cubic EXTENSIONS OF GENERAL FIELDS}

In this section $L / K$ is a cubic extension and $V$ is a vector space of finite dimension $n$ over $L$. We fix elements $\eta, \zeta \in L$ such that $1, \eta, \zeta$ form a basis of $L$ over $K$.

5.1. Structure theorems. The subspaces considered in the following lemma will serve as a building block later on. It will be convenient to refer to them as simple triangular subspaces of $V$.

Lemma 5.1. For a subspace $W \in\left[\begin{array}{l}V \\ 3\end{array}\right]_{K}$, the following are equivalent:

(i) $\operatorname{dim}_{L} L W=2$ and every nonzero vector in $W$ has type 1 in $W$.

(ii) $W=\langle x, y, \eta x+\zeta y\rangle_{K}$ for some L-independent vectors $x$ and $y$.

Proof. (ii) $\Rightarrow$ (i) Let $W$ be as in (ii). Clearly $\operatorname{dim}_{L} L W=2$. Suppose two nonzero vectors in $W$ are proportional over $L$, and say

$$
\lambda(a x+b y+c(\eta x+\zeta y))=a^{\prime} x+b^{\prime} y+c^{\prime}(\eta x+\zeta y)
$$


for some $(a, b, c),\left(a^{\prime}, b^{\prime}, c^{\prime}\right) \in K^{3}-\{(0,0,0)\}, \lambda \in L^{\times}$. We have to show $\lambda \in K$. Taking the coefficients of $x$ and $y$ on both sides, we obtain

$$
\left\{\begin{array}{l}
\lambda(a+c \eta)=a^{\prime}+c^{\prime} \eta \\
\lambda(b+c \zeta)=b^{\prime}+c^{\prime} \zeta
\end{array}\right.
$$

and by cross-multiplying,

$$
\lambda(a+c \eta)\left(b^{\prime}+c^{\prime} \zeta\right)=\lambda\left(a^{\prime}+c^{\prime} \eta\right)(b+c \zeta),
$$

i.e.,

$$
a b^{\prime}+c b^{\prime} \eta+a c^{\prime} \zeta=a^{\prime} b+c^{\prime} b \eta+a^{\prime} c \zeta .
$$

Since $1, \eta, \zeta$ is a basis of $L$ over $K$, we have

$$
\left\{\begin{aligned}
b^{\prime}-c^{\prime} b & =0 \\
a c^{\prime}-a^{\prime} c & =0
\end{aligned}\right.
$$

i.e., $b+c \zeta$ and $b^{\prime}+c^{\prime} \zeta$ are linearly dependent over $K$ and the same is true for $a+c \eta$ and $a^{\prime}+c^{\prime} \eta$. Finally, since at least one of $a+c \eta$ and $b+c \zeta$ is nonzero, we must have $\lambda \in K$.

(i) $\Rightarrow$ (ii) Let $W$ be as in (i). First notice that $W \cap \zeta W=0$ (because $W$ contains no vector of type $\geq 2$ ) and $\operatorname{dim}_{K} W=\operatorname{dim}_{K} \zeta W=\frac{1}{2} \operatorname{dim}_{K} L W$, which implies $L W=W \oplus \zeta W$. So if $x$ is any nonzero vector in $W$, the vector $\eta x \in L W$ can be expressed as $\eta x=z-\zeta y$ for some (unique) $y, z \in W$. To show that $x$ and $y$ are $L$-independent, suppose $y=\lambda x$ for some $\lambda \in L$. Since $W$ contains no vector of type $\geq 2, \lambda \in K$. Hence $(\eta+\lambda \zeta) x=z \in W$, which is impossible because $\eta+\lambda \zeta \notin K$. Therefore $x$ and $y$ are $L$-independent, and the three vectors $x, y, z=\eta x+\zeta y$ are $K$-independent and form a basis of $W$ over $K$.

Theorem 5.2. Let $W \in\left[\begin{array}{l}V \\ { }^{*}\end{array}\right]_{K}$ with $\operatorname{comp}_{L} W=0$ and let

$$
W_{2}=\left\langle v \in W \mid \operatorname{type}_{W}(v)=2\right\rangle_{K} .
$$

(i) There are $L$-independent vectors $e_{1}, \ldots, e_{r}$ of $W$ such that

$$
W_{2}=\bigoplus_{i=1}^{r}\left\langle e_{i}, \eta e_{i}\right\rangle_{K}
$$

$$
\left(r=0 \text { if } W_{2}=0\right) \text {. In particular, } \operatorname{dim}_{K} W_{2} \text { is even. }
$$

(ii) $W \cap \eta W$ is a $K$-substructure and $W_{2}=(W \cap \eta W) \oplus\left(W \cap \eta^{-1} W\right)$.

(iii) $\operatorname{dim}_{K}(W \cap \eta W)=\frac{1}{2} \operatorname{dim}_{K} W_{2}$.

Proof. (a) To prove (i), let $\left\{e_{1}, \ldots, e_{r}\right\}$ be a maximal set of $L$-independent vectors of type 2 in $W$. Replacing each $e_{i}$ by $\lambda_{i} e_{i}$ for some scalar $\lambda_{i} \in L^{\times}$, we may assume by Corollary 3.6 that $L e_{i} \cap W=\left\langle e_{i}, \eta e_{i}\right\rangle_{K}$. Let $Z$ be the $K$-substructure $\left\langle e_{1}, \ldots, e_{r}\right\rangle_{K}$. Thus far we know $W_{2} \geq Z \oplus \eta Z$. To prove equality, it remains to show that every vector of type 2 in $W$ is contained in $Z \oplus \eta Z$. Let $v$ be such a vector. By maximality of $r, v$ must belong to $L Z=Z \oplus \eta Z \oplus \zeta Z$, say $v=u_{1}+\eta u_{2}+\zeta u_{3}$ for some $u_{1}, u_{2}, u_{3} \in Z$. Then 
$\zeta u_{3}=v-u_{1}-\eta u_{2} \in W$ and also $u_{3}, \eta u_{3} \in W$. Since $\operatorname{comp}_{L} W=0, u_{3}$ must be zero, i.e., $v \in Z \oplus \eta Z$.

(b) To prove (ii) and (iii), first notice that if $\lambda \in L-K$, then any nonzero vector in $W \cap \lambda W$ is of type 2 in $W$. So $W \cap \eta W \leq W_{2}$ and $W \cap \eta^{-1} W \leq W_{2}$. Therefore $W \cap \eta W=\eta\left(W \cap \eta^{-1} W\right) \leq \eta W_{2}$ and hence $W \cap \eta W \leq W_{2} \cap \eta W_{2}$. The reverse inclusion is trivial; so $W \cap \eta W=W_{2} \cap \eta W_{2}$. Since $1, \eta, \eta^{2}$ is a basis of $L$ over $K$, we have

$$
\begin{aligned}
W \cap \eta W & =W_{2} \cap \eta W_{2}=\bigoplus_{i=1}^{r}\left(\left\langle e_{i}, \eta e_{i}\right\rangle_{K} \cap\left\langle\eta e_{1}, \eta^{2} e_{i}\right\rangle_{K}\right) \\
& =\left\langle\eta e_{1}, \ldots, \eta e_{r}\right\rangle_{K} .
\end{aligned}
$$

Also

$$
W \cap \eta^{-1} W=\eta^{-1}(W \cap \eta W)=\left\langle e_{1}, \ldots, e_{r}\right\rangle_{K} .
$$

(ii) and (iii) now follow immediately.

We can now state the general structure theorem for $K$-subspaces of $V$.

Theorem 5.3. Let $W \in\left[\begin{array}{l}V \\ *\end{array}\right]_{K}$. Then there exist nonnegative integers $\alpha, r, s$, $t$ satisfying

$$
\alpha+r+2 s+t \leq n
$$

and there exist $L$-independent vectors $v_{1}, \ldots, v_{\alpha} ; e_{1}, \ldots, e_{r} ; f_{1}^{\prime}, f_{1}^{\prime \prime}, \ldots$, $f_{s}^{\prime}, f_{s}^{\prime \prime} ; g_{1}, \ldots, g_{t}$ such that $W$ admits the direct sum decomposition over $L$

$$
W=W_{1} \oplus_{L} W_{2} \oplus_{L} W_{3} \oplus_{L} W_{4}
$$

with

$$
\begin{aligned}
& W_{1}=\left\langle v_{1}, \ldots, v_{\alpha}\right\rangle_{L}, \\
& W_{2}=\bigoplus_{i=1}^{r}\left\langle e_{i}, \eta e_{i}\right\rangle_{K}, \\
& W_{3}=\bigoplus_{i=1}^{s}\left\langle f_{i}^{\prime}, f_{i}^{\prime \prime}, \eta f_{i}^{\prime}+\zeta f_{i}^{\prime \prime}\right\rangle_{K}, \\
& W_{4}=\left\langle g_{1}, \ldots, g_{t}\right\rangle_{K} .
\end{aligned}
$$

The subspaces $W_{2}, W_{3}, W_{4}$ are not unique, but the flag $\left(U_{1}, U_{2}, U_{3}, U_{4}\right)$ with $U_{j}=\bigoplus_{i=1}^{j} W_{i}$ is completely determined by $W$. Specifically, $U_{1}=\operatorname{comp}_{L} W$; $U_{2}=\left\langle v \in W \mid \operatorname{type}_{W}(v) \geq 2\right\rangle_{K} ; \quad U_{3}$ is the sum of $U_{2}$ and of all simple triangular subspaces of $W$; and $U_{4}=W$. So the integers $\alpha, r, s, t$ are also uniquely determined by $W$.

Proof. (a) We first show the uniqueness of the flag $\left(U_{1}, U_{2}, U_{3}, U_{4}\right)$. Let $W$ be given by (5) for some choice of $L$-independent vectors $v_{1}, \ldots, g_{t}$. Clearly $\operatorname{comp}_{L} W=W_{1}=U_{1}$. By Lemmas 3.4 and 5.1 every nonzero vector in $W_{3} \oplus W_{4}$ 
has type 1 in $W$ and

$$
\left\langle v \in W \mid \operatorname{type}_{W}(v) \geq 2\right\rangle_{K}=W_{1} \oplus W_{2}=U_{2} .
$$

Let $\tilde{U}_{3}$ denote the sum of $U_{2}$ and of all simple triangular subspaces of $W$. Clearly $U_{3} \leq \widetilde{U}_{3}$. To show the reverse inclusion, let $\langle x, y, \eta x+\zeta y\rangle_{K}$ be a simple triangular subspace of $W$. We have to show $\langle x, y, \eta x+\zeta y\rangle_{K} \leq U_{3}$. Let $\pi$ be the $L$-linear projection of $L W$ onto $L W_{4}$ corresponding to the direct sum decomposition $L W=L U_{3} \oplus L W_{4}$, so that $\pi(W)=W_{4}$. Since $U_{3}=W \cap L U_{3}$, we have only to show $x, y \in L U_{3}$, i.e., $\pi(x)=\pi(y)=0$. But $\eta x+\zeta y \in W$ implies $\eta \pi(x)+\zeta \pi(y)=\pi(\eta x+\zeta y) \in W_{4}$. Since $W_{4}$ is a $K$-substructure, $L W_{4}=W_{4} \oplus \eta W_{4} \oplus \zeta W_{4}$, and this forces $\pi(x)=\pi(y)=0$. Finally $U_{4}=W$ is clear.

(b) To show the existence of the decomposition, let $W_{1}=\operatorname{comp}_{L} W, \alpha=$ $\operatorname{dim}_{L} W_{1}$, and pick a basis $v_{1}, \ldots, v_{\alpha}$ of $W_{1}$ over $L$. Proposition 3.3 gives us a subspace $W^{\prime}$ such that $W=W_{1} \oplus_{L} W^{\prime}$. Since $\operatorname{comp}_{L} W^{\prime}=0$, we may assume without loss of generality that $\operatorname{comp}_{L} W=0$.

(c) Take the second summand to be $W_{2}=\langle v \in W|$ type $\left._{W}(v)=2\right\rangle_{K}$. By Theorem 5.2 there exist $L$-independent vectors $e_{1}, \ldots, e_{r}$ such that

$$
W_{2}=\bigoplus_{i=1}^{r}\left\langle e_{i}, \eta e_{i}\right\rangle_{K} .
$$

(d) For the remaining summands, we first choose a $K$-substructure $Z$ contained in $W$ such that $L W=L W_{2} \oplus L Z$. If $W=W_{2} \oplus Z$, we are done with $W_{3}=0, W_{4}=Z$. Otherwise we can find $K$-independent vectors $x_{1}, \ldots, x_{s}$ such that

$$
W=W_{2} \oplus Z \oplus\left\langle x_{1}, \ldots, x_{s}\right\rangle_{K}
$$

In general $W_{2}$ and $Z \oplus\left\langle x_{1}, \ldots, x_{s}\right\rangle_{K}$ will not form a direct sum over $L$ and we have to rectify this. Adding to each $x_{i}$ an element of $W_{2} \oplus Z$, we may assume that each $x_{i}$ is of the form

$$
x_{i}=\eta u_{i}^{\prime}+\zeta\left(u_{i}^{\prime \prime}+w_{i}\right)
$$

for some $u_{i}^{\prime}, u_{i}^{\prime \prime} \in Z, w_{i} \in\left\langle e_{1}, \ldots, e_{r}\right\rangle_{K}$. We first show that the vectors $u_{1}^{\prime}, u_{1}^{\prime \prime}, \ldots, u_{s}^{\prime}, u_{s}^{\prime \prime}$ are independent (over $K$ or $L$ is the same here). Let

$$
Z_{1}=\left\langle u_{i}^{\prime}, u_{i}^{\prime \prime} \mid i=1, \ldots, s\right\rangle_{K} \leq Z
$$

and $\mu=\operatorname{dim}_{K} Z_{1}$, and consider the subspace

$$
W^{\prime}=W_{2} \oplus Z_{1} \oplus\left\langle x_{1}, \ldots, x_{s}\right\rangle_{K} \leq W .
$$

We have $\operatorname{dim}_{K} W^{\prime}=2 r+\mu+s$ and, since $L W^{\prime}=L W_{2} \oplus L Z_{1}, \operatorname{dim}_{K} L W^{\prime}=$ $3 r+3 \mu$. Since $\operatorname{comp}_{L} W^{\prime}=0$, Theorem 5.2(iii) applies to give

$$
\operatorname{dim}_{K}\left(W^{\prime} \cap \eta W^{\prime}\right)=\frac{1}{2} \operatorname{dim}_{K} W_{2}=r .
$$


From the inequality

$$
\operatorname{dim}_{K}\left(W^{\prime} \cap \eta W^{\prime}\right) \geq \operatorname{dim}_{K} W^{\prime}+\operatorname{dim}_{K} \eta W^{\prime}-\operatorname{dim}_{K} L W^{\prime},
$$

one gets $r \geq 2(2 r+\mu+s)-(3 r+3 \mu)$ or $\mu \geq 2 s$. Therefore all the vectors $u_{i}^{\prime}$ and $u_{i}^{\prime \prime}$ are linearly independent. Now complete the vectors $u_{i}^{\prime}$ and $u_{i}^{\prime \prime}$ into a basis $\left\{u_{1}^{\prime}, \ldots, u_{s}^{\prime} ; u_{1}^{\prime \prime}, \ldots, u_{s}^{\prime \prime} ; g_{1}, \ldots, g_{t}\right\}$ of $Z$ and let $f_{i}^{\prime}=u_{i}^{\prime}$, $f_{i}^{\prime \prime}=u_{i}^{\prime \prime}+w_{i}(i=1, \ldots, s)$. The vectors $e_{1}, \ldots, e_{r} ; f_{1}^{\prime}, f_{1}^{\prime \prime}, \ldots, f_{s}^{\prime}, f_{s}^{\prime \prime}$; $g_{1}, \ldots, g_{t}$ are $L$-independent and we have the direct sum decomposition over $L$

with

$$
W=W_{2} \oplus_{L} W_{3} \oplus_{L} W_{4}
$$

$$
W_{3}=\bigoplus_{i=1}^{s}\left\langle f_{i}^{\prime}, f_{i}^{\prime \prime}, \eta f_{i}^{\prime}+\zeta f_{i}^{\prime \prime}\right\rangle_{K}
$$

and

$$
W_{4}=\left\langle g_{1}, \ldots, g_{t}\right\rangle_{K}
$$

Keeping the notation of Theorem 5.3, we define the type of $W$ to be the quadruple of nonnegative integers

$$
\operatorname{type}(W)=(\alpha, r, s, t)
$$

and the flag of $W$ to be the flag of subspaces

$$
\operatorname{flag}(W)=\left(U_{1}, U_{2}, U_{3}, W\right) .
$$

We have the analogue of Corollary 4.3: the four parameters of type $(W)$ form a complete set of invariants for the action of $\mathrm{GL}_{L}(V)$ on $\left[\begin{array}{l}V \\ *\end{array}\right]_{K}$.

Corollary 5.4. Two $K$-subspaces of $V$ are in the same orbit for $\mathrm{GL}_{L}(V)$ if and only if they have the same type.

Remark (Packing Problem). Theorem 5.3 allows us to solve the following problem:

Given an integer $j \in[0,3]$, find the maximum dimension $d_{j}$ of a $K$-subspace $W$ of $V$ with the property that any vector of $W$ has type at most $j$ in $W$.

The solution is given by

$$
d_{0}=0, \quad d_{1}=\lfloor 3 n / 2\rfloor, \quad d_{2}=2 n, \quad d_{3}=3 n .
$$

Remark. If type $(W)=(\alpha, r, s, t)$ and $\operatorname{flag}(W)=\left(U_{1}, U_{2}, U_{3}, W\right)$, then

$$
\begin{array}{ll}
\operatorname{dim}_{K} U_{1}=3 \alpha, & \operatorname{dim}_{L} U_{1}=\alpha, \\
\operatorname{dim}_{K} U_{2}=3 \alpha+2 r, & \operatorname{dim}_{L} L U_{2}=\alpha+r, \\
\operatorname{dim}_{K} U_{3}=3 \alpha+2 r+3 s, & \operatorname{dim}_{L} L U_{3}=\alpha+r+2 s, \\
\operatorname{dim}_{K} W=3 \alpha+2 r+3 s+t, & \operatorname{dim}_{L} L W=\alpha+r+2 s+t .
\end{array}
$$


The relations above involving $U_{1}, U_{2}$ and $W$ are enough to determine $s$ without having to know $U_{3}$ explicitly, which is sometimes hard to achieve in specific examples.

A necessary and sufficient condition for a quadruple $(\alpha, r, s, t) \in \mathrm{N}^{4}$ to be the type of some $K$-subspace of $V$ is that

$$
\alpha+r+2 s+t \leq n .
$$

Moreover, it is easy to check that given $(\alpha, r, s, t)$ and $\left(\alpha^{\prime}, r^{\prime}, s^{\prime}, t^{\prime}\right)$ quadruples in $\mathrm{N}^{4}$ satisfying (6), there exist subspaces $W, W^{\prime} \in\left[\begin{array}{l}V \\ *\end{array}\right]_{K}$ such that type $(W)=(\alpha, r, s, t)$, type $\left(W^{\prime}\right)=\left(\alpha^{\prime}, r^{\prime}, s^{\prime}, t^{\prime}\right)$ and $W \leq W^{\prime}$ if and only if the following four inequalities hold:

$$
\begin{aligned}
\alpha & \leq \alpha^{\prime}, \\
\alpha+r & \leq \alpha^{\prime}+r^{\prime}, \\
\alpha+r+2 s & \leq \alpha^{\prime}+r^{\prime}+2 s^{\prime}, \\
\alpha+r+2 s+t & \leq \alpha^{\prime}+r^{\prime}+2 s^{\prime}+t^{\prime} .
\end{aligned}
$$

(Use Corollary 3.6 to check that a subspace of type $(0,2,0,0)$ contains a subspace of type $(0,0,1,0)$.) We have now obtained a concrete description of the quotient poset $\left[{ }^{V}\right]_{K} / \mathrm{GL}_{L}(V)$. It is isomorphic to the set of quadruples of nonnegative integers satisfying (6), with the order relation given by (7). However, it will be more convenient to work with an equivalent description of this quotient poset in terms of a modification of the notion of partition, which we now proceed to develop.

5.2. The poset of modified partitions. A modified partition is a quadruple $\left(\lambda_{1}, \lambda_{2}, \lambda_{3} \mid \lambda_{4}\right)$ of nonnegative integers satisfying $\lambda_{1} \geq \lambda_{2} \geq \lambda_{3}$ and $\lambda_{4} \leq$ $\left(\lambda_{1}-\lambda_{2}\right) / 2$; note that in a modified partition the terms to the left of the vertical bar form an ordinary partition with at most three parts. We write $\mathscr{P}_{n}$ for the set of modified partitions with largest part $\lambda_{1} \leq n$.

To each quadruple $(\alpha, r, s, t) \in \mathrm{N}^{4}$, associate the modified partition $\left(\lambda_{1}\right.$, $\left.\lambda_{2}, \lambda_{3} \mid \lambda_{4}\right)$ defined by

$$
\lambda_{1}=\alpha+r+2 s+t, \quad \lambda_{2}=\alpha+r, \quad \lambda_{3}=\alpha, \quad \lambda_{4}=s .
$$

This defines a bijection between the set of quadruples satisfying (6) and $\mathscr{P}_{n}$, by which we can transport to $\mathscr{P}_{n}$ the order relation on quadruples defined by (7). The order relation on $\mathscr{P}_{n}$ is thus defined by

$$
\left(\lambda_{1}, \lambda_{2}, \lambda_{3} \mid \lambda_{4}\right) \leq\left(\mu_{1}, \mu_{2}, \mu_{3} \mid \mu_{4}\right)
$$

if $\lambda_{i} \leq \mu_{i}(i=1,2,3)$ and $\lambda_{2}+2 \lambda_{4} \leq \mu_{2}+2 \mu_{4}$, i.e., $\left(\lambda_{1}, \lambda_{2}, \lambda_{3}\right) \leq\left(\mu_{1}, \mu_{2}\right.$, $\mu_{3}$ ) as ordinary partitions and $\lambda_{2}+2 \lambda_{4} \leq \mu_{2}+2 \mu_{4}$.

Define the diagram $D_{\lambda}$ of a modified partition $\lambda=\left(\lambda_{1}, \lambda_{2}, \lambda_{3} \mid \lambda_{4}\right)$ as the set of unit squares making up the Ferrers diagram of the usual partition $\left(\lambda_{1}, \lambda_{2}, \lambda_{3}\right)$, to which have been adjoined $2 \lambda_{4}$ triangular 'saw-teeth' of unit 


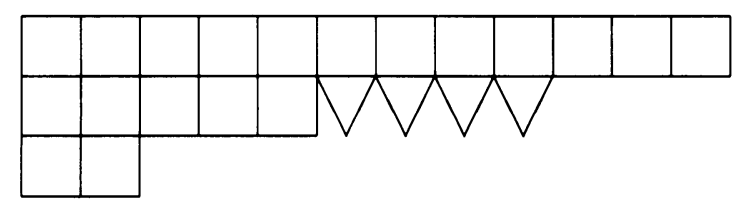

FIGURE 1 . The diagram of $\lambda=(12,5,2 \mid 2)$

height and base, hanging down from the portion of $\lambda_{1}$ overhanging $\lambda_{2}$ and left-justified against $\lambda_{2}$. See Figure 1 for an example of diagram.

The order relation between modified partitions corresponds to containment of diagrams: for $\lambda, \mu \in \mathscr{P}_{n}$,

$$
\lambda \leq \mu \Leftrightarrow D_{\lambda} \subseteq D_{\mu} .
$$

Define the weight of $\lambda$ by

$$
|\lambda|=\lambda_{1}+\lambda_{2}+\lambda_{3}+\lambda_{4},
$$

which equals the area of the diagram $D_{\lambda}$.

We can now state the main result of $\S 5$.

Theorem 5.5. Let $L / K$ be a cubic extension of fields and let $V$ be a vector space of finite dimension $n$ over $L$. The quotient poset $\left[{ }^{V}\right]_{K} / \mathrm{GL}_{L}(V)$ is independent of the fields $K$ and $L$. It is isomorphic to the poset $\mathscr{P}_{n}$ of modified partitions introduced above.

Next follows additional information we have gathered about the poset $\mathscr{P}_{n}$. It is ranked and Peck, of height $3 n$, with minimum element the empty partition $(0,0,0 \mid 0)$, maximum element $(n, n, n \mid 0)$; the rank of a modified partition equals its weight. It is not a lattice: the two elements $(3,2,0 \mid 0)$ and $(3,1,0 \mid$ 1) of rank 5 both cover the two elements $(3,1,0 \mid 0)$ and $(3,0,0 \mid 1)$ of rank 4. Let $a_{m}(n)$ denote the size of the $m$ th level of the poset and let $g(N ; M)$ be the number of (ordinary) partitions of $M$ inside a $3 \times N$ rectangle (or 0 if $M$ or $N$ is negative). Since any modified partition with $2 s$ saw teeth contained in a $3 \times n$ rectangle can be obtained from an ordinary partition contained in a $3 \times(n-2 s)$ rectangle by inserting $2 s$ columns each consisting of a box in the first row and a saw tooth in the second row, we have

$$
a_{m}(n)=\sum_{s \geq 0} g(n-2 s ; m-3 s) .
$$

The numbers $a_{m}(n)$ have the generating function

$$
\sum_{n, m \geq 0} a_{m}(n) x^{n} q^{m}=\frac{1}{(x ; q)_{4}\left(1-x^{2} q^{3}\right)} .
$$


The special case $q=1$ gives

$$
\sum_{n=0}^{\infty}\left|\mathscr{P}_{n}\right| x^{n}=\frac{1}{(1-x)^{5}(1+x)},
$$

from which one can obtain the cardinality of the quotient poset:

$$
\begin{aligned}
\left|\mathscr{P}_{n}\right| & =\frac{1}{96}\left(2 n^{4}+24 n^{3}+100 n^{2}+168 n+93\right)+\frac{1}{32}(-1)^{n} \\
& =\frac{1}{48}(n+1)(n+3)^{2}(n+5)+ \begin{cases}0 & \text { for } n \text { odd }, \\
1 / 16 & \text { for } n \text { even }\end{cases} \\
& \sim \frac{1}{48} n^{4} \quad \text { as } n \rightarrow \infty .
\end{aligned}
$$

Remark. It is easily checked that the antiautomorphism of Theorem 2.3 is the involution that sends the type $(\alpha, r, s, t)$ to $(n-(\alpha+r+2 s+t), t, s, r)$ or, equivalently, that sends the modified partition $\left(\lambda_{1}, \lambda_{2}, \lambda_{3} \mid \lambda_{4}\right)$ to $\left(n-\lambda_{3}\right.$, $\left.n-\lambda_{2}-2 \lambda_{4}, n-\lambda_{1} \mid \lambda_{4}\right)$.

5.3. Triangular subspaces. In preparation to Section 6 , we take a closer look at $K$-subspaces of $V$ containing no vectors of type $\geq 2$. A $K$-subspace of $V$ of type $(0,0, s, 0)$ for some $s$ will be called triangular. Recall that the simple triangular subspaces are those of type $(0,0,1,0)$. Suppose $W \in\left[\begin{array}{l}V \\ *\end{array}\right]_{K}$ contains no vector of type $\geq 2$, i.e., type $(W)=(0,0, s, t)$ for some integers $s, t$. For such a subspace $W$, we let

$$
\mathscr{T}(W) \text { and } \mathscr{T}_{i}(W)
$$

denote respectively the set of triangular subspaces of $W$ and the set of such subspaces of type $(0,0, i, 0), i=0, \ldots, s$. We order $\mathscr{T}(W)$ by inclusion and will show in Proposition 5.9 that $\mathscr{T}(W)$ is in fact a sublattice of $\left[{ }^{W}\right]_{K}$; we will see that $\mathscr{T}(W)$ is a ranked poset, with its $i$ th level equal to $\mathscr{T}_{i}(W)$. The subspace $U_{3}$ defined by flag $(W)=\left(0,0, U_{3}, W\right)$ will be called the triangular component of $W$, written $\mathrm{TC}(W)$. Theorem 5.3 implies that $\mathrm{TC}(W)$ is the largest element of $\mathscr{T}(W)$.

Proposition 5.6. Let $W$ be a triangular subspace of $V$.

(i) $L W=W \oplus \lambda W$ for all $\lambda \in L-K$.

(ii) Given any vector $x \in W$, there is a unique vector $y \in W$ such that $\eta x+\zeta y \in W$. Moreover, if $x \neq 0$, then $x$ and $y$ are L-independent and $\langle x, y, \eta x+\zeta y\rangle_{K}$ is the unique simple triangular subspace of $W$ containing $x$.

We can rephrase (ii) by saying that the collection of simple triangular subspaces of $W$ constitutes a spread of $W$.

Proof. Same as (i) $\Rightarrow$ (ii) of Lemma 5.1.

Here are two direct consequences of the previous proposition. 
Corollary 5.7. If $W \in\left[\begin{array}{l}V \\ *\end{array}\right]_{K}$ contains no vector of type $\geq 2$, then a nonzero vector of $W$ belongs to the triangular component of $W$ if and only if it belongs to a (necessarily unique) simple triangular subspace of $W$.

Corollary 5.8. Suppose $V=L W$ with $W$ simple triangular. Then the group $\operatorname{Stab}_{V}(W)$ acts regularly on the set of nonzero vectors of $W$.

Remark. Suppose $W_{1}, W_{2} \in\left[\begin{array}{l}V \\ *\end{array}\right]_{K}$ contain no vector of type $\geq 2$. Then $\mathrm{TC}\left(W_{1} \cap W_{2}\right) \leq \mathrm{TC}\left(W_{1}\right) \cap \mathrm{TC}\left(W_{2}\right)$, with no equality in general. However, equality holds if $W_{1}$ and $W_{2}$ are both contained in a common $K$-subspace of $V$ containing no vector of type $\geq 2$, as will follow from the next proposition.

Proposition 5.9. Suppose $W \in\left[\begin{array}{l}V \\ *\end{array}\right]_{K}$ contains no vector of type $\geq 2$. Then $\mathscr{T}(W)$ is a sublattice of $\left[{ }^{W}\right]_{K}$. More precisely, if $W_{i} \in \mathscr{T}_{s_{i}}(W), i=1,2$, then $W_{1} \cap W_{2} \in \mathscr{T}_{s_{3}}(W)$ and $W_{1}+W_{2} \in \mathscr{T}_{s_{4}}(W)$ for some $s_{3}, s_{4}$ satisfying $s_{1}+s_{2}=s_{3}+s_{4}$.

Proof. Let $W_{1}, W_{2} \in \mathscr{T}(W)$. We first show $W_{1} \cap W_{2} \in \mathscr{T}(W)$. Let $0 \neq x \in$ $W_{1} \cap W_{2}$. By Corollary 5.7, the unique subspace $U \in \mathscr{T}_{1}(W)$ containing $x$ is actually contained in $W_{1}$ and in $W_{2}$. Then $x \in U \leq W_{1} \cap W_{2}$ implies $x \in$ $\operatorname{TC}\left(W_{1} \cap W_{2}\right)$. Hence $W_{1} \cap W_{2}=\mathrm{TC}\left(W_{1} \cap W_{2}\right)$. To show $W_{1}+W_{2} \in \mathscr{T}(W)$, it suffices to notice that $W_{1}, W_{2} \in \mathscr{T}(W)$ implies $W_{1}+W_{2} \leq \mathrm{TC}\left(W_{1}+W_{2}\right) \leq$ $W_{1}+W_{2}$. Finally, the assertions about type follow by a simple counting of dimensions.

Corollary 5.10. Suppose $W \in\left[\begin{array}{c}V \\ *\end{array}\right]_{K}$ contains no vector of type $\geq 2$. If $W_{1}, W_{2} \in$ $\left[{ }^{W}\right]_{K}$, then $\mathrm{TC}\left(W_{1} \cap W_{2}\right)=\mathrm{TC}\left(W_{1}\right) \cap \mathrm{TC}\left(W_{2}\right)$.

Proof. Direct consequence of $\mathrm{TC}\left(W_{1} \cap W_{2}\right) \leq \mathrm{TC}\left(W_{1}\right) \cap \mathrm{TC}\left(W_{2}\right) \leq W_{1} \cap W_{2}$ and the fact that $\operatorname{TC}\left(W_{1}\right) \cap \mathrm{TC}\left(W_{2}\right)$ is triangular.

Corollary 5.11. Suppose $W \in\left[{ }^{V}\right]_{K}$ contains no vector of type $\geq 2$. Let $W_{1}, W_{2}$ $\in \mathscr{T}(W)$. If $W_{1} \cap W_{2}=0$, then $L W_{1} \cap L W_{2}=0$.

Proof. Let type $\left(W_{i}\right)=\left(0,0, s_{i}, 0\right), \quad i=1,2$. We know type $\left(W_{1}+W_{2}\right)=$ $\left(0,0, s_{1}+s_{2}, 0\right)$. Since $L W_{1}+L W_{2}=L\left(W_{1}+W_{2}\right)$, we have

$$
\begin{aligned}
\operatorname{dim}_{L}\left(L W_{1} \cap L W_{2}\right) & =\operatorname{dim}_{L} L W_{1}+\operatorname{dim}_{L} L W_{2}-\operatorname{dim}_{L} L\left(W_{1}+W_{2}\right) \\
& =2 s_{1}+2 s_{2}-2\left(s_{1}+s_{2}\right)=0
\end{aligned}
$$

and the result follows.

Lemma 5.12. Suppose $W \in\left[\begin{array}{l}V \\ { }^{*}\end{array}\right]_{K}$ contains no vector of type $\geq 2$. Let $W_{1} \in$ $\mathscr{T}(W)$ and $U \in \mathscr{T}_{1}(W)^{\prime}$. Then one of the following holds:

(i) $U \leq W_{1}$.

(ii) $L U \cap L W_{1}=0$.

Proof. By Proposition 5.9, $U \cap W_{1}$ is triangular. Since the only subspaces in $\mathscr{T}(U)$ are $U$ and 0 , we have either $U \cap W_{1}=U$, i.e., $U \leq W_{1}$; or $U \cap W_{1}=0$, which implies $L U \cap L W_{1}=0$ by Corollary 5.11. 
Proposition 5.13. Suppose $W \in\left[\begin{array}{l}V \\ { }_{*}\end{array}\right]_{K}$ contains no vector of type $\geq 2$. For any $W_{1} \in \mathscr{T}(W)$, there exists a subspace $W_{2} \in\left[\begin{array}{l}W \\ { }^{*}\end{array}\right]_{K}$ such that $W=W_{1} \oplus_{L} W_{2}$.

Proof. By Theorem 5.3, TC $(W)$ is a direct summand of $W$ over $L$ and $W_{1} \leq$ $\mathrm{TC}(W)$. So we may assume without loss of generality that $W$ is of triangular type. An induction argument using Lemma 5.12 now gives the result.

Let $W$ be a triangular subspace of $V$. We now know that $\mathscr{T}(W)$ is a ranked poset, where the rank of an element $W_{1} \in \mathscr{T}(W)$ equals $\frac{1}{3} \operatorname{dim}_{K} W_{1}$. The group $\operatorname{Stab}_{V}(W)$ acts by automorphisms on the poset $\mathscr{T}(W)$. Using Theorem 5.3 and Proposition 5.13, it is easy to check the following.

Proposition 5.14. Let $W$ be a triangular subspace of $V$. The action of the group $\operatorname{Stab}_{V}(W)$ on the poset $\mathscr{T}(W)$ is transitive on every level $\mathscr{T}_{i}(W)$.

Remark. We conjecture that if $W$ is a triangular subspace of $V$, say of type $(0,0, s, 0)$, then the poset $\mathscr{T}(W)$ is isomorphic to $\left[{ }^{s}{ }^{s}\right]_{L}$. We have checked this in some special cases, and it also agrees with the enumerative results of Section 6.1.

5.4. Linear maps. We now examine the behavior of the type and the flag of a subspace with respect to $L$-linear maps. First some notation. If $\mathscr{F}_{i}=$ $\left(U_{1}^{(i)}, U_{2}^{(i)}, U_{3}^{(i)}, U_{4}^{(i)}\right), i=1,2$, are two flags of $K$-subspaces of $V$, we write $\mathscr{F}_{1} \leq \mathscr{F}_{2}$ when $U_{j}^{(1)} \leq U_{j}^{(2)}$ for all $j$. So if $W_{1}, W_{2} \in\left[\begin{array}{l}V \\ *\end{array}\right]_{K}$ and $W_{1} \leq W_{2}$, then flag $\left(W_{1}\right) \leq \operatorname{flag}\left(W_{2}\right)$. If $\mathscr{F}=\left(U_{1}, \ldots, U_{4}\right)$ is a flag and $T \in \operatorname{End}_{L}(V)$, we also write $T(\mathscr{F})=\left(T\left(U_{1}\right), \ldots, T\left(U_{4}\right)\right)$.

If $x \in W \in\left[\begin{array}{l}V \\ *\end{array}\right]_{K}$ and $T \in \mathrm{GL}_{L}(V)$, then $\operatorname{type}_{T(W)}(T x)=\operatorname{type}_{W}(x)$, type $(T(W))=\operatorname{type}(W)$, and flag $(T(W))=T(\operatorname{flag}(W))$. If $T$ is not invertible, the situation is more complicated. The following lemma is trivial to check.

Lemma 5.15. Let $x \in W \in\left[\begin{array}{l}V \\ *\end{array}\right]_{K}$ and let $T \in \operatorname{End}_{L}(V)$. If $T(x) \neq 0$, then $\operatorname{type}_{T(W)}(T x) \geq \operatorname{type}_{W}(x)$.

Lemma 5.16. Let $W \in\left[\begin{array}{l}V \\ *\end{array}\right]_{K}$ and $T \in \operatorname{End}_{L}(V)$ with $T(W) \neq 0$. Then:

$$
\begin{aligned}
& \operatorname{type}(W)=(1,0,0,0) \Rightarrow \operatorname{type}(T(W))=(1,0,0,0), \\
& \operatorname{type}(W)=(0,1,0,0) \Rightarrow \operatorname{type}(T(W))=(0,1,0,0), \\
& \operatorname{type}(W)=(0,0,1,0) \Rightarrow \operatorname{type}(T(W))=(1,0,0,0) \\
& \quad \text { or }(0,1,0,0) \text { or }(0,0,1,0), \\
& \operatorname{type}(W)=(0,0,0,1) \Rightarrow \operatorname{type}(T(W))=(0,0,0,1) .
\end{aligned}
$$

Proof. The cases type $(W)=(1,0,0,0),(0,1,0,0),(0,0,0,1)$ are clear. For the case type $(W)=(0,0,1,0)$, let $W=\langle x, y, \eta x+\zeta y\rangle_{K}$. If $T x$ and $T y$ are $L$-independent, then $T(W)$ has type $(0,0,1,0)$. Assume now $T x$ and $T y$ are dependent over $L$, so that $T(W)$ is contained in some subspace $U \in\left[\begin{array}{l}V \\ 1\end{array}\right]_{L}$. If $T(W)=U$, then $\operatorname{type}(W)=(1,0,0,0)$. (It is easy to check that this case does occur.) Otherwise $\left.\operatorname{ker} T\right|_{W} \neq 0$. Using Corollary 5.8, we 
may assume without loss of generality that $T x=0$. Since $T(W) \neq 0$, we must have $T y \neq 0$; therefore $T(W)=\langle T y, \zeta T y\rangle_{K}$ has type $(0,1,0,0)$.

As a direct consequence of Lemma 5.16 and Theorem 5.3, we obtain the following.

Proposition 5.17. Let $W \in\left[\begin{array}{l}V \\ *\end{array}\right]_{K}$ and $T \in \operatorname{End}_{L}(V)$. Then

$$
T(\operatorname{flag}(W)) \leq \operatorname{flag}(T(W)) .
$$

More generally, if $T(W) \leq W_{1} \in\left[\begin{array}{l}V \\ *\end{array}\right]_{K}$, then $T(\operatorname{flag}(W)) \leq$ flag $\left(W_{1}\right)$.

Remark. In general, type $(W)$ and type $(T(W))$ are incomparable. For example a subspace of type $(0,0,0, t)$ can be sent to ariy $K$-subspace of $V$ of dimension not exceeding $t$ by some $L$-linear endomorphism of $V$.

We conclude $\$ 5.4$ with two results that will be useful for the enumeration in $\S 6$.

Proposition 5.18. Let $W \in\left[\begin{array}{l}V \\ *\end{array}\right]_{K}$ have type $(0, r, 0,0)$ for some $r$ and suppose $L W=V$. Let $Z=W \cap \eta W$, which is a $K$-structure of $V$. For $T \in \operatorname{End}_{L}(V)$, we have

$$
T(W) \leq W \Leftrightarrow T(Z) \leq Z .
$$

In particular, $\operatorname{Stab}_{V}(W)=\operatorname{Stab}_{V}(Z) \cong \mathrm{GL}_{K}(Z)$.

Proof. Use Theorem 5.2 and Proposition 3.8.

Proposition 5.19. Let $W, W^{\prime}$ be two $K$-subspaces of $V$ of respective types $(0,0, s, 0)$ and $(0, r, 0,0)$. Then the $K$-subspace of $\operatorname{Hom}_{L}\left(L W, L W^{\prime}\right)$ consisting of those maps sending $W$ into $W^{\prime}$ has dimension 3 rs over $K$.

Proof. Since a triangular subspace is the direct sum over $L$ of simple triangular subspaces, it is enough to consider the case $s=1$; so suppose $W=$ $\left\langle x_{1}, y_{1}, \eta x_{1}+\zeta y_{1}\right\rangle_{K}$ for some $L$-independent vectors $x_{1}$ and $y_{1}$. Since any $T \in \operatorname{Hom}_{L}\left(L W, L W^{\prime}\right)$ is completely determined by the images $x=T x_{1}$ and $y=T y_{1}$, there is a $K$-linear isomorphism between the subspace we are considering and the subspace $U=\left\{(x, y) \in W^{\prime} \times W^{\prime} \mid \eta x+\zeta y \in W^{\prime}\right\}$. We have $U=\varphi^{-1}\left(W^{\prime}\right)$, where $\varphi: W^{\prime} \times W^{\prime} \rightarrow L W^{\prime}$ is defined by $\varphi(x, y)=\eta x+\zeta y$. The fact that $\eta$ and $\zeta$ are $K$-independent implies $\eta W^{\prime}+\zeta W^{\prime}=L W^{\prime}$, i.e., $\varphi$ is surjective. Consequently, $\operatorname{dim}_{K} \operatorname{ker} \varphi=4 r-3 r=r$ and $\operatorname{dim}_{K} U=$ $\operatorname{dim}_{K} W^{\prime}+\operatorname{dim}_{K} \operatorname{ker} \varphi=2 r+r=3 r$.

\section{CUBiC EXTENSIONS OF FINITE FIELDS}

In this section we work with the finite fields $K=\mathbf{F}_{q} \leq L=\mathbf{F}_{q^{3}}$, where $q$ is a power of a prime; $V$ is vector space of dimension $n$ over $L$. Our main result is Theorem 6.3 which counts the number of $K$-subspaces of $V$ of a given type. 
6.1. Counting triangular subspaces. We begin with some enumerative results concerning triangular subspaces.

Proposition 6.1. Let $W \in\left[{ }^{V}{ }^{*}\right]_{K}$ be of type $(0,0, s, 0)$. Let $i, j, k$ be nonnegative integers satisfying $i+j \leq s$ and $k \leq i$. Let $W_{0} \in \mathscr{T}_{j}(W)$ and $W_{1} \in \mathscr{T}_{k}(W)$ with $W_{0} \cap W_{1}=0$. Then

$$
\mid\left\{W_{2} \in \mathscr{T}_{i}(W) \mid W_{2} \cap W_{0}=0 \text { and } W_{2} \geq W_{1}\right\} \mid=q^{3 j(i-k)}\left[\begin{array}{c}
s-j-k \\
i-k
\end{array}\right]_{q^{3}} .
$$

In particular,

and

$$
\begin{gathered}
\left|\left\{W_{2} \in \mathscr{T}_{i}(W) \mid W_{2} \cap W_{0}=0\right\}\right|=q^{3 i j}\left[\begin{array}{c}
s-j \\
i
\end{array}\right]_{q^{3}}, \\
\left|\left\{W_{2} \in \mathscr{T}_{i}(W) \mid W_{2} \geq W_{1}\right\}\right|=\left[\begin{array}{c}
s-k \\
i-k
\end{array}\right]_{q^{3}}
\end{gathered}
$$

$$
\left|\mathscr{T}_{i}(W)\right|=\left[\begin{array}{l}
S \\
i
\end{array}\right]_{q^{3}} .
$$

Proof. Let $\beta$ denote the number we want to compute. For each $x \in W-\{0\}$, let $U(x)$ be the unique simple triangular subspace of $W$ containing $x$, as given by Proposition 5.6. Count tuples $\left(W_{2} ; x_{1}, \ldots, x_{i-k}\right)$ such that $W_{2} \in \mathscr{T}_{i}(W)$, $W_{2} \cap W_{0}=0, W_{2} \geq W_{1}$ and $x_{1}, \ldots, x_{i-k}$ are nonzero vectors satisfying

$$
W_{2}=W_{1} \oplus_{L} \bigoplus_{l=1}^{i-k} U\left(x_{l}\right)
$$

in two different ways: choosing $W_{2}$ first or choosing $x_{1}, \ldots, x_{i-k}$ first. Using Corollary 5.11 and Lemma 5.12 it is easy to see that

$$
\begin{aligned}
& \beta\left(q^{3 i}-q^{3 k}\right)\left(q^{3 i}-q^{3(k+1)}\right) \cdots\left(q^{3 i}-q^{3(i-1)}\right) \\
& \quad=\left(q^{3 s}-q^{3(j+k)}\right)\left(q^{3 s}-q^{3(j+k+1)}\right) \cdots\left(q^{3 s}-q^{3(j+i-1)}\right),
\end{aligned}
$$

from which the result follows.

Proposition 6.2. Suppose $V=L W$ with $W \in\left[\begin{array}{l}V \\ *\end{array}\right]_{K}$ of type $(0,0, s, 0)$. Then

$$
\left|\operatorname{Stab}_{V}(W)\right|=\left|\mathrm{GL}\left(s, q^{3}\right)\right|=\prod_{i=0}^{s-1}\left(q^{3 s}-q^{3 i}\right) .
$$

Proof. We proceed by induction on $s$. For $s=0$ there is nothing to prove. For $s=1$, Corollary 5.8 implies that $\left|\operatorname{Stab}_{V}(W)\right|$ equals the number of nonzero vectors of $W$, i.e., $q^{3}-1$. For $s>1$, assume the result true for $s-1$. Using Propositions 5.13 and 5.14, it is easy to see that $\operatorname{Stab}_{V}(W)$ acts transitively on the set of pairs $\left(W_{1}, U\right) \in \mathscr{T}_{s-1}(W) \times \mathscr{T}_{1}(W)$ such that $W=W_{1} \oplus U$. The number of such pairs is $\left[\begin{array}{c}s \\ s-1\end{array}\right]_{q^{3}} q^{3 s-3}$ by Proposition 6.1. Let us now examine the stabilizer $H$ of a fixed such pair $\left(W_{1}, U\right)$ under the $\operatorname{action}$ of $\operatorname{Stab}_{V}(W)$. 
If we write $V_{1}=L W_{1}$ and $V_{2}=L U$, any $T \in H$ is completely determined by its restrictions to $V_{1}$ and $V_{2}$. Using induction and the case $s=1$, we get

$$
\begin{aligned}
|H| & =\left|\operatorname{Stab}_{V_{2}}(U)\right| \cdot\left|\operatorname{Stab}_{V_{1}}(W)\right| \\
& =\left(q^{3}-1\right) \prod_{i=0}^{s-2}\left(q^{3 s-3}-q^{3 i}\right)
\end{aligned}
$$

and therefore

$$
\left|\operatorname{Stab}_{V}(W)\right|=\frac{\left(q^{3 s}-1\right)}{\left(q^{3}-1\right)} q^{3 s-3} \cdot|H|=\prod_{i=0}^{s-1}\left(q^{3 s}-q^{3 i}\right) .
$$

Remark. We have preferred to use an elementary approach to obtain the cardinality of $\operatorname{Stab}_{V}(W)$. But we can actually prove for general cubic extensions that, under the hypotheses of Proposition 6.2, $\operatorname{Stab}_{V}(W) \cong \mathrm{GL}(s, L)$. Details will appear later.

6.2. Counting subspaces of a given type. We now determine the sizes of the orbits for the action of $\mathrm{GL}_{L}(V)$ on $\left[{ }^{V}\right]_{K}$, i.e., we compute the number of $K$-subspaces of $V$ of a given type.

Let $W \in\left[{ }^{V}{ }^{*}\right]_{K}$ be of type $(\alpha, r, s, t)$ and put $c=n-(\alpha+r+2 s+t)$. We first compute the order of the group $\operatorname{Stab}_{V}(W)$. Suppose $W$ admits the decomposition

$$
W=W_{1} \oplus_{L} W_{2} \oplus_{L} W_{3} \oplus_{L} W_{4}
$$

given by Theorem 5.3 and let $W_{5}=0$. Put $V_{i}=L W_{i}(i=1, \ldots, 4)$ and choose an $L$-subspace $V_{5}$ of $V$ such that

$$
V=V_{1} \oplus V_{2} \oplus V_{3} \oplus V_{4} \oplus V_{5} .
$$

Let $\pi_{i}: V \rightarrow V_{i}(i=1, \ldots, 5)$ denote the corresponding $L$-linear projections; so $\pi_{i}(W)=W_{i}$ for all $i$.

If $T \in \operatorname{Stab}_{V}(W)$, then $T(\operatorname{flag}(W))=\operatorname{flag}(W)$. So the matrix of $T$ with respect to suitable bases of the $V_{i}$ (in the order $V_{1}, \ldots, V_{5}$ ) has the parabolic form

$$
\left[\begin{array}{ccccc}
T_{1} & T_{12} & T_{13} & T_{14} & T_{15} \\
0 & T_{2} & T_{23} & T_{24} & T_{25} \\
0 & 0 & T_{3} & T_{34} & T_{35} \\
0 & 0 & 0 & T_{4} & T_{45} \\
0 & 0 & 0 & 0 & T_{5}
\end{array}\right]
$$

where $T_{i}=\left.\pi_{i} \circ T\right|_{V_{i}}$ for $i=1, \ldots, 5$ and $T_{i j}=\left.\pi_{i} \circ T\right|_{V_{j}}$ for $1 \leq i<j \leq 5$. For each $i, T_{i} \in \mathrm{GL}_{L}\left(V_{i}\right)$ and $T_{i}\left(W_{i}\right)=W_{i}$ (because $\left.\pi_{i}(W)=W_{i}\right)$, i.e., $T_{i} \in \operatorname{Stab}_{V_{i}}\left(W_{i}\right)$. For each $i<j, T_{i j} \in \operatorname{Hom}_{L}\left(V_{j}, V_{i}\right)$ and $T_{i j}\left(W_{j}\right) \leq W_{i}$. Conversely, for any choice of maps $T_{i} \in \operatorname{Stab}_{V_{i}}\left(W_{i}\right)$ and $T_{i j} \in \operatorname{Hom}_{L}\left(V_{j}, V_{i}\right)$ satisfying $T_{i j}\left(W_{j}\right) \leq W_{i}$, the corresponding $T \in \mathrm{GL}_{L}(V)$ given by (8) belongs 
to $\operatorname{Stab}_{V}(W)$. So to determine the order of $\operatorname{Stab}_{V}(W)$, it suffices to determine separately for each $i$ and $j$ the order of $\operatorname{Stab}_{V_{i}}\left(W_{i}\right)$ and the number of maps $T_{i j}$ as above. For convenience, we will use the notation

$$
\begin{gathered}
\beta_{i}=\left|\operatorname{Stab}_{V_{i}}\left(W_{i}\right)\right|, \\
\beta_{i j}=\left|\left\{T_{i j} \in \operatorname{Hom}_{L}\left(V_{j}, V_{i}\right) \mid T_{i j}\left(W_{j}\right) \leq W_{i}\right\}\right|
\end{gathered}
$$

and

$$
\exp _{q}(a)=q^{a}
$$

We examine each case separately, starting with the diagonal blocks.

(a) Counting $T_{1}: \operatorname{Stab}_{V_{1}}\left(W_{1}\right)=\operatorname{Stab}_{V_{1}}\left(V_{1}\right)=\mathrm{GL}_{L}\left(V_{1}\right)$; so $\beta_{1}=\left|\mathrm{GL}\left(\alpha, q^{3}\right)\right|$.

(b) Counting $T_{2}$ : By Proposition 5.18, $\beta_{2}=|\mathrm{GL}(r, q)|$.

(c) Counting $T_{3}$ : By Proposition 6.2, $\beta_{3}=\left|\mathrm{GL}\left(s, q^{3}\right)\right|$.

(d) Counting $T_{4}$ : By Proposition 3.8, $\beta_{4}=|\mathrm{GL}(t, q)|$.

(e) Counting $T_{5}: \operatorname{Stab}_{V_{5}}\left(W_{5}\right)=\operatorname{Stab}_{V_{5}}(0)=\mathrm{GL}_{L}\left(V_{5}\right)$; so $\beta_{5}=\left|\mathrm{GL}\left(c, q^{3}\right)\right|$.

(f) Counting $T_{1 j}(j=2, \ldots, 5)$ : Since $W_{1}=V_{1}$, the condition $T_{1 j}\left(W_{j}\right) \leq$ $W_{1}$ is satisfied for all $T_{1 j} \in \operatorname{Hom}_{L}\left(V_{j}, V_{1}\right)$; so $\beta_{1 j}=\left|\operatorname{Hom}_{L}\left(V_{j}, V_{1}\right)\right|=$ $\exp _{q}\left(3 \alpha \operatorname{dim}_{L} V_{j}\right)$.

(g) Counting $T_{i 5}(i=1, \ldots, 4)$ : Since $W_{5}=0$, the condition $T_{i 5}\left(W_{5}\right) \leq W_{i}$ is satisfied for all $T_{i 5} \in \operatorname{Hom}_{L}\left(V_{5}, V_{i}\right)$; so

$$
\beta_{i 5}=\left|\operatorname{Hom}_{L}\left(V_{5}, V_{i}\right)\right|=\exp _{q}\left(3 c \operatorname{dim}_{L} V_{i}\right) .
$$

(h) Counting $T_{23}$ : By Proposition 5.19, $\beta_{23}=q^{3 r s}$.

(i) Counting $T_{i 4}(i=1,2,3)$ : By Proposition 3.8(a), $\beta_{i 4}=\exp _{q}\left(t \operatorname{dim}_{K} W_{i}\right)$.

The situation is summarized in the following table.

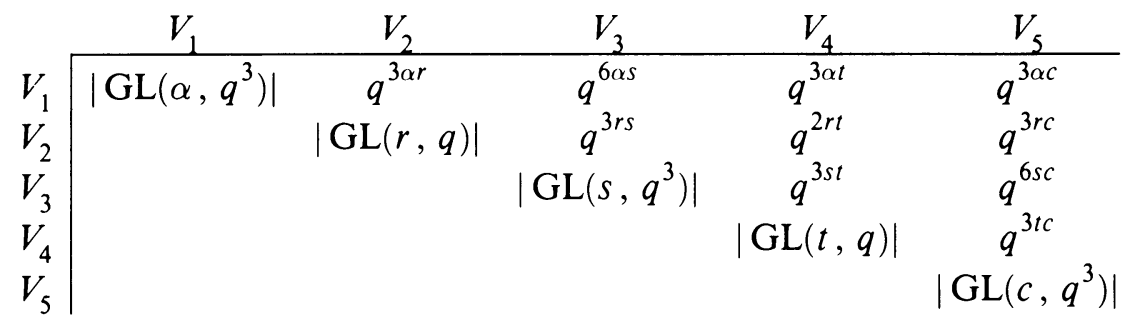

Using the fact that

$$
|\mathrm{GL}(m, q)|=\prod_{i=0}^{m-1}\left(q^{m}-q^{i}\right)=(-1)^{m} q^{\left(\begin{array}{c}
m \\
2
\end{array}\right)}(q ; q)_{m},
$$

we compute

$$
\begin{aligned}
\left|\operatorname{Stab}_{V}(W)\right|= & (-1)^{n-s}\left(q^{3} ; q^{3}\right)_{\alpha}\left(q^{3} ; q^{3}\right)_{s}\left(q^{3} ; q^{3}\right)_{c}(q ; q)_{r}(q ; q)_{t} \\
& \cdot \exp _{q}\left\{3\left(\begin{array}{l}
n \\
2
\end{array}\right)-2\left(\begin{array}{c}
r \\
2
\end{array}\right)-2\left(\begin{array}{l}
t \\
2
\end{array}\right)-r t-\left(\begin{array}{c}
3 s \\
2
\end{array}\right)-3 s(r+t)\right\} .
\end{aligned}
$$


A short computation using the fact that the number of subspaces $W \in\left[\begin{array}{l}V \\ *\end{array}\right]_{K}$ of type $(\alpha, r, s, t)$ equals $\left|\mathrm{GL}_{L}(V)\right| /\left|\operatorname{Stab}_{V}(W)\right|$ for $\operatorname{type}(W)=(\alpha, r, s, t)$ gives the following theorem.

Theorem 6.3. Let $K=\mathbf{F}_{q} \leq L=\mathbf{F}_{q^{3}}$ and let $V$ be a vector space of dimension $n$ over $L$. If $\alpha, r, s, t$ are nonnegative integers satisfying $\alpha+r+2 s+t \leq n$, the number of subspaces $W \in\left[\begin{array}{l}V \\ *\end{array}\right]_{K}$ of type $(\alpha, r, s, t)$ is given by

$$
\begin{aligned}
f(\alpha, r, s, t ; n, q)= & \frac{(-1)^{s}\left(q^{3} ; q^{3}\right)_{n} \exp _{q}\left\{2\left(\begin{array}{c}
r \\
2
\end{array}\right)+2\left(\begin{array}{c}
t \\
2
\end{array}\right)+r t+\left(\begin{array}{c}
3 s \\
2
\end{array}\right)+3 s r+t\right\}}{\left(q^{3} ; q^{3}\right)_{\alpha}\left(q^{3} ; q^{3}\right)_{s}\left(q^{3} ; q^{3}\right)_{c}(q ; q)_{r}(q ; q)_{t}} \\
= & {\left[\begin{array}{c}
n \\
\alpha, r, 2 s, t, c
\end{array}\right]_{q^{3}}\left[\begin{array}{c}
2 s \\
s
\end{array}\right]_{q^{3}} \frac{\left(q^{3} ; q^{3}\right)_{r}}{(q ; q)_{r}} \frac{\left(q^{3} ; q^{3}\right)_{t}}{(q ; q)_{t}}(-1)^{s}\left(q^{3} ; q^{3}\right)_{s} } \\
& \cdot \exp _{q}\left\{2\left(\begin{array}{c}
r \\
2
\end{array}\right)+2\left(\begin{array}{c}
t \\
2
\end{array}\right)+r t+\left(\begin{array}{c}
3 s \\
2
\end{array}\right)+3 s(r+t)\right\}
\end{aligned}
$$

where $c=n-(\alpha+r+2 s+t)$.

Notice that $f(\alpha, r, s, t ; n, q)$ is a polynomial in $q$ with integral coefficients, and it is symmetric in $r$ and $t$. If $s=0$, the coefficients are all nonnegative; but this is not the case for general $s$. We have

$$
\lim _{q \rightarrow 1} f(\alpha, r, s, t ; n, q)=\left\{\begin{array}{cc}
\left(\begin{array}{c}
n \\
\alpha, r, t, c
\end{array}\right) 3^{r+t} & \text { if } s=0 \\
0 & \text { if } s>0
\end{array}\right.
$$

6.3. Some $q$-series identities. We are going to apply Theorem 6.3 to obtain $q$-analogues of some transformation and evaluation formulas for hypergeometric series. We first recall the standard notation for hypergeometric and basic hypergeometric functions.

$$
{ }_{p} F_{m}\left(\begin{array}{c}
a_{1}, \ldots, a_{p} \\
b_{1}, \ldots, b_{m}
\end{array} \mid x\right)=\sum_{i=0}^{\infty} \frac{\left(a_{1}\right)_{i} \cdots\left(a_{p}\right)_{i} x^{i}}{\left(b_{1}\right)_{i} \cdots\left(b_{m}\right)_{i} i !}
$$

where $(a)_{i}=\Gamma(a+i) / \Gamma(a)$ is the shifted factorial, and

$$
{ }_{p} \phi_{m}\left(\begin{array}{c}
a_{1}, \ldots, a_{p} \\
b_{1}, \ldots, b_{m}
\end{array} \mid q ; x\right)=\sum_{i=0}^{\infty} \frac{\left(a_{1} ; q\right)_{i} \cdots\left(a_{p} ; q\right)_{i} x^{i}}{\left(b_{1} ; q\right)_{i} \cdots\left(b_{m} ; q\right)_{i}(q ; q)_{i}}
$$

with the $q$-shifted factorials $(a ; q)_{i}$ as defined in $\S 4.2$.

We will also need to know the number of $K$-subspaces $W$ of $V$ of a given dimension satisfying $L W=V$. This number can be computed when $L / K$ is an extension of finite fields of arbitrary degree.

Theorem 6.4. Let $K=\mathbf{F}_{q} \leq L=\mathbf{F}_{q^{d}}$ where $q$ is a power of a prime, and let $V$ be a vector space of finite dimension $n$ over $L$. Writing

$$
c_{n}(m)=c_{n}(m ; q, d)=\left|\left\{W \in\left[\begin{array}{l}
V \\
m
\end{array}\right]_{K} \mid L W=V\right\}\right|
$$


for $m$ nonnegative integer, we have

$$
\begin{aligned}
c_{n}(m) & =\sum_{i=0}^{n}(-1)^{i}\left[\begin{array}{l}
n \\
i
\end{array}\right]_{q^{d}}\left[\begin{array}{c}
d(n-i) \\
m
\end{array}\right]_{q} q^{d\left(\begin{array}{l}
i \\
2
\end{array}\right)} \\
& =\left[\begin{array}{c}
d n \\
m
\end{array}\right]_{q}{ }_{d} \phi_{d-1}\left(\begin{array}{c}
q^{-d n+m}, q^{-d n+m+1}, \ldots, q^{-d n+m+d-1} \\
q^{-d n+1}, q^{-d n+2}, \ldots, q^{-d n+d-1}
\end{array} \mid q^{d} ; q^{d(n-m)}\right),
\end{aligned}
$$

which is a polynomial in $q$ with integral coefficients. Moreover, $c_{n}(m) \neq 0$ if and only if $n \leq m \leq d n$.

Proof. Use Möbius inversion on the poset $\mathscr{P}=\left[\begin{array}{l}V \\ *\end{array}\right]_{L}$. The Möbius function of $\mathscr{P}$ is given by $\mu(Y, U)=(-1)^{j} q^{d\left(\begin{array}{l}j \\ 2\end{array}\right)}$ with $j=\operatorname{dim}_{L} U-\operatorname{dim}_{L} Y$ for $Y, U \in \mathscr{P}, \quad Y \leq U$. For $Y \in \mathscr{P}$, define

$$
f_{=}(Y)=\left|\left\{W \in\left[\begin{array}{c}
Y \\
m
\end{array}\right]_{K} \mid L W=Y\right\}\right|,
$$

so that the number we want to compute is $f_{=}(V)$. For $U \in \mathscr{P}$, let

$$
f_{\leq}(U)=\sum_{\substack{Y \in \mathscr{P} \\ Y \leq U}} f_{=}(Y),
$$

so that $f_{\leq}(U)=\left[\begin{array}{c}d i \\ m\end{array}\right]_{q}$ if $\operatorname{dim}_{L} U=i$. By Möbius inversion,

$$
\begin{aligned}
f_{=}(V) & =\sum_{U \in \mathscr{P}} \mu(U, V) f_{\leq}(U) \\
& =\sum_{i=0}^{n}\left[\begin{array}{c}
n \\
i
\end{array}\right]_{q^{d}}(-1)^{n-i} q^{d\left(\begin{array}{c}
n-i \\
2
\end{array}\right)}\left[\begin{array}{c}
d i \\
m
\end{array}\right]_{q} \\
& =\sum_{i=0}^{n}(-1)^{i}\left[\begin{array}{c}
n \\
i
\end{array}\right]_{q^{d}}\left[\begin{array}{c}
d(n-i) \\
m
\end{array}\right]_{q} q^{d\left(\begin{array}{c}
i \\
2
\end{array}\right)} .
\end{aligned}
$$

Standard manipulations transform this expression to the basic hypergeometric form stated above. The remaining assertions are trivial to check.

Remark. Fix $m \in \mathbf{N}$ and let $N \in \mathbf{N}$ with $N \geq m$. Let $U$ be a vector space of dimension $N$ over $L=\mathbf{F}_{q^{d}}$. Counting all pairs $(W, Y) \in\left[\begin{array}{l}U \\ *\end{array}\right]_{K} \times\left[\begin{array}{l}U \\ *\end{array}\right]_{L}$ with $L W=Y$ in two different ways gives

$$
\left[\begin{array}{c}
d N \\
m
\end{array}\right]_{q}=\sum_{n=0}^{m} c_{n}(m)\left[\begin{array}{c}
N \\
n
\end{array}\right]_{q^{d}} .
$$

Putting $x=q^{d N}$ we obtain

$$
\frac{\left(x ; q^{-1}\right)_{m}}{(q ; q)_{m}}=\sum_{n=0}^{m} c_{n}(m) \frac{\left(x ; q^{-d}\right)_{n}}{\left(q^{d} ; q^{d}\right)_{n}} .
$$

Each member of this identity is a polynomial in $x$ of degree $m$; since this identity is satisfied for infinitely many values of $x$, it must hold for all val- 
ues of $x$. So the numbers $c_{n}(m ; q, d)$ (multiplied by the normalizing factors $\left.(q ; q)_{m} /\left(q^{d} ; q^{d}\right)_{n}\right)$ are the connection coefficients between $\left\{\left(x ; q^{-1}\right)_{i}\right\}_{i \geq 0}$ and $\left\{\left(x ; q^{-d}\right)_{i}\right\}_{i \geq 0}$, bases of the vector space $R[x]$ of polynomials in $x$ with coefficients in, say, the field $R=\mathrm{Q}(q)$ of rational functions in $q$ with coefficients in $\mathbf{Q}$.

Returning to the case of a cubic extension, we have

$$
\begin{aligned}
c_{n}(m) & =c_{n}(m ; q, 3) \\
& =\left[\begin{array}{c}
3 n \\
m
\end{array}\right]_{q}{ }_{3} \phi_{2}\left(\begin{array}{c}
q^{-3 n+m}, q^{-3 n+m+1}, q^{-3 n+m+2} \\
q^{-3 n+1}, q^{-3 n+2}
\end{array} \mid q^{3} ; q^{3(n-m)}\right) .
\end{aligned}
$$

On the other hand, if we group the $K$-subspaces $W$ of $V$ satisfying $L W=V$ according to their type, we see that

$$
c_{n}(m)=\sum f(\alpha, r, s, t ; n, q),
$$

where the sum extends to all quadruples $(\alpha, r, s, t) \in \mathrm{N}^{4}$ such that

$$
\alpha+r+2 s+t=n \quad \text { and } 3 \alpha+2 r+3 s+t=m .
$$

It will be more convenient to make a change of parameter. Put

$$
M=3 n-m,
$$

so that (9) becomes

$$
c_{n}(m)=\left[\begin{array}{c}
3 n \\
M
\end{array}\right]_{q}{ }_{3} \phi_{2}\left(\begin{array}{c}
q^{-M}, q^{-M+1}, q^{-M+2} \\
q^{-3 n+1}, q^{-3 n+2}
\end{array} \mid q^{3} ; q^{3(M-2 n)}\right)
$$

and the nonvanishing condition $n \leq m \leq 3 n$ becomes $0 \leq M \leq 2 n$. Solving for $\alpha$ and $r$ in terms of $s$ and $t$ in (11) gives

$$
\alpha=n-M+s+t \quad \text { and } \quad r=M-3 s+2 t .
$$

We restrict ourselves to the case $n \geq M$, so that the term $f(\alpha, r, s, t ; n, q)$ corresponding to $s=t=0$ is nonzero. Equation (10) becomes

$$
c_{n}(m)=\sum_{s, t \geq 0} f(n-M+s+t, M-3 s-2 t, s, t ; n, q) .
$$

One computes

$$
\begin{gathered}
f(n-M+s+t, M-3 s-2 t, s, t ; n, q)=\frac{(-1)^{M} q^{3 n M-\left(\begin{array}{c}
M \\
2
\end{array}\right)}\left(q^{-3 n} ; q^{3}\right)_{M}}{(q ; q)_{M}} \\
\cdot \frac{\left(q^{-M} ; q\right)_{3 s} q^{3 s}}{\left(q^{3} ; q^{3}\right)_{s}\left(q^{3(n-M+1)} ; q^{3}\right)_{s}} \cdot \frac{\left(q^{3 s-M} ; q\right)_{2 t} q^{t(t+2-M)}}{(q ; q)_{t}\left(q^{3(n-M+s+1)} ; q^{3}\right)_{t}} .
\end{gathered}
$$


Putting (12) and (13) together and simplifying by $(-1)^{M} q^{3 n M-\left(\begin{array}{c}M \\ 2\end{array}\right)} /(q ; q)_{M}$ gives

$$
\begin{aligned}
& \left(q^{-3 n} ; q\right)_{M 3} \phi_{2}\left(\begin{array}{c|c}
q^{-M}, q^{-M+1}, q^{-M+2} \\
q^{-3 n+1}, q^{-3 n+2} & q^{3} ; q^{3 M-6 n}
\end{array}\right) \\
& =\left(q^{-3 n} ; q^{3}\right)_{M} \sum_{s \geq 0} \frac{\left(q^{-M} ; q\right)_{3 s} q^{3 s}}{\left(q^{3} ; q^{3}\right)_{s}\left(q^{3(n-M+1)} ; q^{3}\right)_{s}} \sum_{t \geq 0} \frac{\left(q^{3 s-M} ; q\right)_{2 t} q^{t(t+2-M)}}{(q ; q)_{t}\left(q^{3(n-M+s+1)} ; q^{3}\right)_{t}} .
\end{aligned}
$$

Both sides of (14) are finite sums with the number of terms depending only on $M$; both sides are rational functions in $q^{3 n}$. Since (14) is valid for infinitely many values of $n$ ( $M$ fixed), we may replace $q^{-3 n}$ by an independent variable $a$. Thus we obtain

Theorem 6.5. Let $M \in \mathbf{N}$ and let $a$ and $q$ be indeterminates. Then

$$
\begin{aligned}
& (a ; q)_{M}{ }_{3} \phi_{2}\left(\begin{array}{c}
q^{-M}, q^{-M+1}, q^{-M+2} \mid \\
a q, a q^{2}
\end{array} \mid q^{3} ; a^{2} q^{3 M}\right) \\
& \quad=\left(a ; q^{3}\right)_{M} \sum_{s \geq 0} \frac{\left(q^{-M} ; q\right)_{3 s} q^{3 s}}{\left(q^{3} ; q^{3}\right)_{s}\left(a^{-1} q^{3-3 M} ; q^{3}\right)_{s}} \sum_{t \geq 0} \frac{\left(q^{3 s-M} ; q\right)_{2 t} q^{t(t+2-M)}}{(q ; q)_{t}\left(a^{-1} q^{3(s-M+1)} ; q^{3}\right)_{t}} .
\end{aligned}
$$

If we change the order of the two summations, we have the equivalent formula

$$
\begin{gathered}
(a ; q)_{M}{ }_{3} \phi_{2}\left(\begin{array}{c}
q^{-M}, q^{-M+1}, q^{-M+2} \\
a q, a q^{2}
\end{array} \mid q^{3} ; a^{2} q^{3 M}\right) \\
=\left(a ; q^{3}\right)_{M} \sum_{t \geq 0} \frac{\left(q^{-M} ; q\right)_{2 t} q^{t(t+2-M)}}{(q ; q)_{t}\left(a^{-1} q^{3-3 M} ; q^{3}\right)_{t}} \\
\cdot{ }_{3} \phi_{2}\left(\begin{array}{c}
q^{2 t-M}, q^{2 t-M+1}, q^{2 t-M+2} \\
0, a^{-1} q^{3(t-M+1)}
\end{array} \mid q^{3} ; q^{3}\right) .
\end{gathered}
$$

The sums in the right-hand sides of both formulas are actually over all $s, t \in \mathbf{N}$ such that $3 s+2 t \leq M$; both sides are polynomials in $a$.

Remark. The limiting case $(q \rightarrow 1)$ of Theorem 6.5 is the "strange" transformation formula of Gessel and Stanton [6, Equation 3.19]

$$
\begin{gathered}
(3 a)_{M}{ }_{3} F_{2}\left(\begin{array}{c|c}
-M / 3,(1-M) / 3,(2-M) / 3 & 1 \\
a+\frac{1}{3}, a+\frac{2}{3} & 1
\end{array}\right) \\
=3^{M}(a)_{M 2} F_{1}\left(\begin{array}{c|c}
-M / 2,(1-M) / 2 & 4 \\
1-a-M & \frac{3}{3}
\end{array}\right) .
\end{gathered}
$$

From Theorem 6.5 we can obtain several "strange" evaluation formulas by specializing the parameter $a$ so that the left-hand side becomes summable. 
For example, by choosing $a=q^{-M-1}, M=3 p+2$ with $p \in \mathbf{N}, p \geq 1$, and using the $q$-analogue of Vandermonde's formula [1, Corollary 2.4], one obtains

$$
\sum_{s \geq 0} \frac{\left(q^{-3 p-2} ; q\right)_{3 s} q^{3 s}}{\left(q^{3} ; q^{3}\right)_{s}\left(q^{-6 p} ; q^{3}\right)_{s}} \sum_{t \geq 0} \frac{\left(q^{3 s-3 p-2} ; q\right)_{2 t} q^{t(t-3 p)}}{(q ; q)_{t}\left(q^{3 s-6 p-1} ; q^{3}\right)_{t}}=0 .
$$

Choosing $a=q^{-M-2}, M=3 p+2$ with $p \in \mathbf{N}$ and using $q$-Vandermonde's formula again gives

$$
\begin{gathered}
\sum_{s \geq 0} \frac{\left(q^{-3 p} ; q\right)_{3 s} q^{3 s}}{\left(q^{3} ; q^{3}\right)_{s}\left(q^{1-6 p} ; q^{3}\right)_{s}} \sum_{t \geq 0} \frac{\left(q^{3 s-3 p-2} ; q\right)_{2 t} q^{t(t-3 p)}}{(q ; q)_{t}\left(q^{3 s-6 p+1} ; q^{3}\right)_{t}} \\
\quad=\frac{\left(q^{-3 p-4} ; q\right)_{3 p+2}\left(q^{-2} ; q^{3}\right)_{p}}{\left(q^{-3 p-4} ; q^{3}\right)_{3 p+2}\left(q^{-3 p-3} ; q^{3}\right)_{p}} .
\end{gathered}
$$

If one puts $a=q^{(3-3 M) / 2}$, one can apply the $q$-analogue of Saalschütz's formula [2, §8.4]. The case $M=3 p, p \in \mathbf{N}$, gives

$$
\begin{gathered}
\sum_{s \geq 0} \frac{\left(q^{-3 p} ; q\right)_{3 s} q^{3 s}}{\left(q^{3} ; q^{3}\right)_{s}\left(q^{3(1-3 p) / 2} ; q^{3}\right)_{s}} \sum_{t \geq 0} \frac{\left(q^{3 s-3 p} ; q\right)_{2 t} q^{t(t+2-3 p)}}{(q ; q)_{t}\left(q^{3 s+3(1-3 p) / 2} ; q^{3}\right)_{t}} \\
\quad=\frac{\left(q^{(3-3 p) / 2} ; q^{3}\right)_{p}\left(q^{(1-3 p) / 2} ; q^{3}\right)_{p}\left(q^{(3-9 p) / 2} ; q\right)_{3 p}}{\left(q^{(5-9 p) / 2} ; q^{3}\right)_{p}\left(q^{(3 p-1) / 2} ; q^{3}\right)_{p}\left(q^{(3-9 p) / 2} ; q^{3}\right)_{3 p}} .
\end{gathered}
$$

There are two similar formulas for the cases $M \equiv 1$ or $2(\bmod 3)$.

Added in proof. The conjecture mentioned after Proposition 5.14 has now been proved.

\section{REFERENCES}

1. G. Andrews, The theory of partitions, Addison-Wesley, Reading, Mass., 1976.

2. W. Bailey, Generalized hypergeometric series, Cambridge Univ. Press, Cambridge, 1935.

3. A. Borel, Linear algebraic groups, Benjamin, New York, 1969.

4. N. Bourbaki, Algèbre, Chapitres 1-3, Diffusion C.C.L.S., Paris, 1970.

5. S. Gelbart, I. Piatetski-Shapiro and S. Rallis, Explicit constructions of automorphic $L$ functions, Lecture Notes in Math., vol. 1254, Springer-Verlag, New York, 1987.

6. I. Gessel and D. Stanton, Strange evaluations of hypergeometric series, Siam J. Math. Anal. 13 (1982), 295-308.

7. D. S. Kim and P. Rabau, Action on Grassmannians associated with commutative semisimple algebras, Trans. Amer. Math. Soc. 326 (1991), 157-168.

8. D. S. Kim and P. Rabau, Field extensions and isotropic subspaces in symplectic geometry, Geom. Dedicata 34 (1990), 281-293.

9. P. Rabau, Enumeration in vector spaces over a field extension, Ph.D. thesis, University of Minnesota, 1988.

10. R. Stanley, Quotients of Peck posets, Order 1 (1984), 29-34.

Department of Mathematics, The Ohio State University, Columbus, Ohio 43210 